\title{
Unsteady flow organization of compressible planar base flows
}

\author{
R. A. Humble, ${ }^{\text {a) }}$ F. Scarano, and B. W. van Oudheusden \\ Faculty of Aerospace Engineering, Delft University of Technology, Kluyverweg 1, 2629 HS, Delft, \\ The Netherlands
}

(Received 15 January 2007; accepted 5 April 2007; published online 12 July 2007)

\begin{abstract}
The unsteady flow features of a series of two-dimensional, planar base flows are examined, within a range of low-supersonic Mach numbers in order to gain a better understanding of the effects of compressibility on the organized global dynamics. Particle image velocimetry is used as the primary diagnostic tool in order to characterize the instantaneous near wake behavior, in combination with data processing using proper orthogonal decomposition. The results show that the mean flowfields are simplified representations of the instantaneous flow organizations. Generally, each test case can be characterized by a predominant global mode, which undergoes an evolution with compressibility, within the Mach number range considered. (The term "global mode" is defined herein as an organized global dynamical behavior of the near wake region, recognizing that the near wake dynamics may be describable in terms of several global modes.) At Mach 1.46, the predominant global mode can be characterized by a sinuous or flapping motion. With increasing compressibility, this flapping mode decreases, and the predominant global mode evolves into a pulsating motion aligned with the wake axis at Mach 2.27. These global modes play an important role in the distributed nature of the turbulence properties. The turbulent mixing processes become increasingly confined to a narrower redeveloping wake with increasing compressibility. Global maximum levels of the streamwise turbulence intensity and the kinematic Reynolds shear stress occur within the vicinity of the mean reattachment location, and show no systematic trend with compressibility. In contrast, the global maximum level of the vertical turbulence intensity moves upstream from the redeveloping wake toward the mean reattachment location. The vertical turbulence intensity decays thereafter more slowly than the other turbulence quantities. Overall, the local maximum levels of the turbulence properties decrease appreciably with increasing compressibility. (C) 2007 American Institute of Physics. [DOI: 10.1063/1.2739411]
\end{abstract}

\section{INTRODUCTION}

The compressible near wake region behind a blunt-based body contains a series of complicated flow phenomena that constitute a fundamental fluid dynamics research problem. The near wake contains a myriad of flow structures, characterized by a large separated flow region, bounded by separated free shear layers and the base wall. Because of its fundamental, as well as practical relevance in a variety of fluid dynamic applications, such as high-speed projectiles and powered missiles, numerous experimental efforts over the decades have sought to improve the physical understanding of its complex behavior. Schlieren and shadowgraph methods have historically been used to characterize shear layer growth rates, determine the location of shock and expansion waves, as well as provide a visualization of the large-scale structures found to be present. Yet these techniques are somewhat unsuited for the accurate determination of turbulent features at high convective Mach numbers (i.e., high compressibility), since they have been shown to become highly three-dimensional in nature. ${ }^{1}$ Planar imaging techniques, such as Mie scattering, have therefore been used, to provide instantaneous structural information on the flow organization of the shear layer, ${ }^{1,2}$ as well as in the near wake

\footnotetext{
${ }^{a)}$ Author to whom correspondence should be addressed. Tel: $+31(0) 15$ 2785169; Fax: +31(0)15 2787077. Electronic mail: r.a.humble@tudelft.nl
}

region. ${ }^{3,4}$ Such studies have provided direct evidence of large-scale structures along the shear layers, and at the wake interface, using spatial covariance analyses to describe their size, eccentricity, and orientation. Yet planar imaging techniques are incapable of providing quantitative turbulence information, which is critically important for a better understanding of the fluid dynamic mechanisms present.

Numerous studies have therefore focused attention on characterizing in detail the mean velocity distribution, turbulence intensities, turbulent kinetic energy, and kinematic Reynolds shear stress distribution on both planar $^{5-7}$ and axisymmetric $^{8-10}$ base flow configurations. The majority of such studies have primarily made use of laser Doppler velocimetry (LDV), with the statistical data being used by the computational community to help validate the most promising computational codes, which have typically made use of both Reynolds-averaged Navier-Stokes (RANS) and large eddy simulation (LES) techniques. ${ }^{11-13}$ Of particular interest in recent years has been the study of the instantaneous flow organization of the near wake region. This has been motivated by the recognized importance of the unsteady motion of the reattachment point and the associated recompression shock wave system, which can lead to fluctuating base pressures, ${ }^{14}$ as well as fluctuating loads on the body itself. Furthermore, it has become increasingly evident that data on the unsteady flow features of compressible base flows may 
be useful for the validation of computational models, since it has been shown that the consideration of the near wake unsteadiness significantly improves the numerical prediction of the average flowfield properties. ${ }^{15}$ Although the use of pointby-point velocity measurements provides valuable mean and turbulence statistics, the inability to make large-scale instantaneous velocity flowfield measurements represents a significant obstacle in obtaining a more thorough description of the near wake global dynamics.

Advances in laser and digital imaging technology have led to rapid developments in nonintrusive, planar flow diagnostic tools, such as particle image velocimetry (PIV) in particular. This technique is capable of making instantaneous whole-field velocity measurements, rendering it suitable to investigate large-scale unsteady flow features. Although PIV has historically found widespread application as a standard diagnostic tool in low-speed incompressible flows, ${ }^{16}$ extending the technique into the high-speed compressible flow regime has become possible with the introduction of highenergy short-pulsed lasers, as well as short interframe transfer CCD cameras. PIV has been successfully applied to a variety of high-speed flow problems of practical interest, such as jets, ${ }^{17}$ mixing layers, ${ }^{18}$ and boundary layers, ${ }^{19}$ making the study of the compressible planar base flow problem well posed.

Although large-scale organized motions are obvious in visualization studies of compressible base flows, it has been difficult to incorporate them into an underlying description of the global dynamics, and to describe the role of these dynamics in the overall distributed nature of the turbulence properties. While there is a general consensus as to the effects of compressibility in turbulent shear flows, such as leading to a reduction in shear layer growth rates, ${ }^{20,21}$ turbulent fluctuating velocities, and shear stresses, ${ }^{22,23}$ as well as large-scale turbulent structures appearing more threedimensional and disorganized than under incompressible conditions, ${ }^{1,24}$ less agreement is found when attempting to formulate a more unified picture of the role of compressibility in the underlying global dynamics. It is desirable then to describe the flowfield in terms of its most dominant features, or processes, and aim to provide a simpler conceptual picture that can be more readily understood. Much attention has therefore been focussed upon the development of low-order or simplified representations of complicated fluid dynamical systems. This has led to interest in statistical techniques such as Proper Orthogonal Decomposition (POD) in particular. This technique has been introduced into fluid mechanics ${ }^{25}$ as a tool to highlight the most prominent coherent motions within turbulent flows, as well as to facilitate the development of a low-order description of the system's overall dynamics. The technique has been successfully applied to a variety of practical flow problems, including boundary layers, ${ }^{26}$ turbulent jets, ${ }^{27}$ as well as compressible flows. ${ }^{28}$ PIV data are particularly suited for POD analyses since the entire spatial velocity field is available, leading to the construction of global eigenmodes.

Previous studies that have attempted to address the organized global dynamics of compressible planar base flows ${ }^{29,30}$ have visualized the large-scale near wake motion, leading to a characterization of the instantaneous near wake behavior. The application of POD to the problem ${ }^{31}$ has led to hypotheses being made regarding the underlying flow organization. Generally, under transonic conditions, the most dynamically significant feature of the flow could be characterized by a sinuous or flapping motion. Under fully supersonic conditions, the near wake could be characterized by a streamwiseoriented pulsating motion. Predominant modes have also been independently documented for flat plate wakes using Schlieren ${ }^{32}$ and holographic interferometry, ${ }^{33}$ as well as axisymmetric cylinders and cones under both subsonic and supersonic conditions using RANS/LES computations. ${ }^{11}$ Yet no systematic study documenting the behavior of these global modes with compressibility has been reported for the planar base flow case, nor have their role in the distributed nature of the turbulence properties been properly addressed.

The present paper aims to study the unsteady flow features of two-dimensional, planar base flows, in order to gain a better understanding of the effects of compressibility on the organized global dynamics of the near wake region, as well as their role in the distributed nature of the turbulence properties. Experiments are carried out at several Mach numbers in the low-supersonic range. PIV is used as the primary diagnostic tool in combination with data processing using POD. Mean and instantaneous velocity measurements are obtained, enabling a visualization of the near wake behavior. Turbulence statistics are then presented, and the role of organized global dynamics in the distributed nature of the turbulence properties is addressed. The velocity fields are then analyzed by POD to make further statements regarding the unsteady flow organization. Anticipating our discussion later on, we first define a key compressibility parameter; the convective Mach number $M_{c}=\Delta U /\left(a_{1}+a_{2}\right)$, where $\Delta U$ is the velocity change across the initial constant-pressure mixing shear layer, and $a_{1}$ and $a_{2}$ are the corresponding speeds of sound. The convective Mach number is considered as an appropriate parameter to scale the effects of compressibility in supersonic shear flows, and even though a single parameter may not be universally applicable, ${ }^{34}$ it is still considered a useful parameter to delineate the effects of compressibility and will be used for this purpose in the present work.

\section{APPARATUS AND EXPERIMENTAL TECHNIQUE}

Experiments were performed in the blow-down transonic-supersonic wind tunnel (TST-27) of the HighSpeed Aerodynamics Laboratories at Delft University of Technology. The facility generates flows within the Mach number range $0.5-4.2$ in a test section of dimensions $280 \mathrm{~mm} \times 270 \mathrm{~mm}$. The Mach number was set by means of a continuous variation of the throat section and flexible nozzle walls. The tunnel operates at unit Reynolds numbers ranging from $30 \times 10^{6}$ to $130 \times 10^{6} \mathrm{~m}^{-1}$, enabling a blowdown operating use of the tunnel of approximately $300 \mathrm{~s}$. The model consisted of a symmetric double-wedge with a sharp leading edge, giving a deflection angle of $7.13^{\circ}$. This was followed by a $30 \mathrm{~mm}$ long thick plate with constant thickness of $h=20 \mathrm{~mm}$. The total chord length was $110 \mathrm{~mm}$. The model terminated with a vertical base and spanned the 
TABLE I. Experimental conditions.

\begin{tabular}{lcccc}
\hline \hline & \multicolumn{3}{c}{ Case } & Uncertainties \\
\cline { 2 - 4 } & & & 3 & $(\%)$ \\
\hline$M_{\infty}$ & 1 & 2 & 2.27 & 2 \\
$M_{c}$ & 1.46 & 1.78 & 1.01 & $<10$ \\
$U_{\infty}(\mathrm{m} / \mathrm{s})$ & 0.65 & 0.84 & 528 & 1 \\
$P_{0}(\mathrm{kPa})$ & 398 & 461 & 255 & 1 \\
$T_{0}(\mathrm{~K})$ & 192 & 194 & 263 & 1 \\
$\mathrm{Re}_{h}, 1 \times 10^{4}$ & 273 & 273 & 62 & 3 \\
\hline \hline
\end{tabular}

entire width of the test section. In the present study, experiments at three different Mach numbers were conducted that are summarized in Table I.

Wall static pressure, plenum stagnation pressure, and stagnation temperature measurements were used to determine the flow conditions. In Table I, the freestream Mach number $M_{\infty}$ corresponds to that along the thick flat plate immediately upstream of the terminating base. A schematic representation of the experimental configuration is shown in Fig. 1. The inset illustrates the reference coordinate system. A characterization of the incoming flow conditions for the range of Mach numbers considered in the present study determined that the undisturbed freestream flow was uniform, with a turbulence level $\sim 1 \%$. The boundary layer thickness $\delta_{99}$ immediately upstream of the base edge was within the range 1-2 mm, within the Mach number range 1.4-3.0, respectively. The maximum streamwise turbulence intensity within the boundary layers was typically of the order $\left\langle u^{\prime}\right\rangle / U_{\infty} \sim 0.15$ (where $\langle\cdot\rangle$ denotes the root-mean-square quantity). In the present study, the average statistical uncertainties (for all three test cases) associated with the limited number of realizations for the mean velocity $\bar{u}$, turbulence intensity $\left\langle u^{\prime}\right\rangle$, and kinematic Reynolds shear stress $\overline{u^{\prime} v^{\prime}}$, were estimated to be approximately $2 \%, 3 \%$, and $25 \%$, respectively, based upon the maximum standard deviation and correlation coefficient measured in each experiment, using a $95 \%$ confidence interval. ${ }^{35}$

Two-component PIV is employed in the present study. Flow seeding constitutes one of the most critical aspects of PIV in high-speed flows. A 2D distributor rake was used to seed a fraction of the flow in the settling chamber with titanium dioxide $\left(\mathrm{TiO}_{2}\right)$ particles with a nominal median diameter of $50 \mathrm{~nm}$ and estimated bulk density of $200 \mathrm{~kg} / \mathrm{m}^{3}$. Hot- wire anemometry measurements performed in the freestream of the facility revealed no noticeable difference in the mean velocity field, and only a $0.2 \%$ increase in turbulence intensity as a result of the seeding device. A previous particle response assessment study has been carried out across an oblique shock wave in the facility under similar experimental conditions to the present study, and returned a particle relaxation time of $\tau_{p}=2.4 \mu \mathrm{s}$, corresponding to a frequency response of $f_{p}=417 \mathrm{kHz}$. ${ }^{6}$ From this relaxation time, it could be inferred that the effective particle size, due to the phenomenon of particle agglomeration, is approximately $400 \mathrm{~nm}$. The seeded streamtube area was approximately $10 \mathrm{~cm}^{2}$ as it entered the center of the test section. The seeded flow was illuminated by a Spectra-Physics Quanta Ray double-pulsed Nd:YAG laser with $400 \mathrm{~mJ}$ pulsed energy and a 6 ns pulse duration at wavelength $532 \mathrm{~nm}$. Laser light tunnel access was provided by a probe inserted into the flow downstream of the model. The laser pulse separation applied in the measurements was $1 \mu \mathrm{s}$, which produced a maximum freestream particle displacement within the range 0.4-0.5 mm. The light sheet was approximately $1 \mathrm{~mm}$ thick.

Images were recorded by a PCO Sensicam QE, a 12-bit Peltier-cooled CCD digital camera with frame-straddling architecture, and a $1376 \times 1040$ pixel sensor. The camera was equipped with a Nikon $60 \mathrm{~mm}$ focal objective with an $f$-number $f_{\#}=8$, which yielded a diffraction limited particle image spacing of approximately 2 pixels. A narrow-bandpass $532 \mathrm{~nm}$ filter was used to suppress background illumination from daylight. All test cases were imaged over a fieldof-view of $70 \mathrm{~mm} \times 54 \mathrm{~mm}$, resulting in a digital resolution of approximately 19.6 pixels $/ \mathrm{mm}$. A dataset size of $400 \mathrm{im}-$ age pairs was acquired in each case at a framing rate of $5 \mathrm{~Hz}$. The recorded images were analyzed using the twodimensional cross-correlation technique WIDIM. ${ }^{36}$ This method is based upon the deformation of correlation windows with an iterative multigrid scheme, which is particularly suited for highly sheared flows. All images were interrogated using square windows of size $31 \times 31$ pixels and an overlap factor of $75 \%$.

\section{POD TECHNIQUE}

The POD is a statistical technique that decomposes a signal into a basis of nonspecified functions chosen to represent the energy of the signal in the fewest number of modes. ${ }^{37}$ In the present study, a decomposition of the time variation of each flowfield into a limited number of modes is

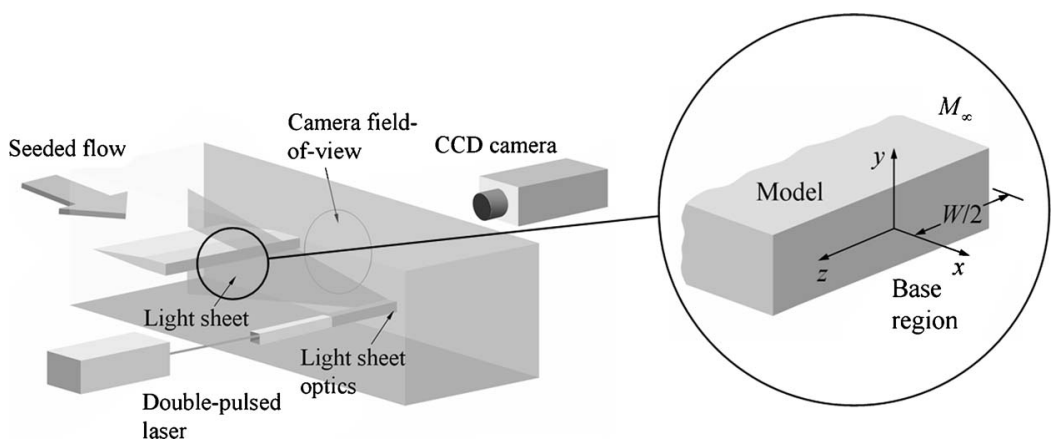

FIG. 1. Schematic representation of the experimental configuration. Reference coordinate system is also shown (inset). 
made. Consider a system in which $N$ data measurements are simultaneously taken at $M$ time instants $t_{n}$, such that the samples are uncorrelated and linearly independent. The data in the present study represent velocity obtained from the PIV study. The mean velocity at a point $\bar{u}(x, y)$ is subtracted from the instantaneous velocity $u\left(x, y, t_{n}\right)$, leaving only data containing fluctuations from the mean $u^{\prime}\left(x, y, t_{n}\right)$. The POD extracts $k$ time-independent orthonormal basis functions, empirical eigenfunctions, or eigenmodes, $\psi_{k}(x, y)$, and timedependent orthonormal amplitude coefficients, $a_{k}\left(t_{n}\right)$, such that the reconstruction

$$
u^{\prime}\left(x, y, t_{n}\right)=\sum_{k=1}^{M} a_{k}\left(t_{n}\right) \psi_{k}(x, y)
$$

is optimal, in the sense that the functions $\psi$ maximize the normalized averaged projection of $\psi$ onto $u^{\prime}$, viz.,

$$
\max _{\psi} \frac{\left\langle\left|\left(u^{\prime}, \psi\right)\right|^{2}\right\rangle}{\|\psi\|^{2}} .
$$

Here, $\|\cdot\|$ denotes the $L^{2}$ norm $\|f\|^{2}=(\cdot, \cdot)$, where $(\cdot, \cdot)$ is the standard Euclidean inner product. $|\cdot|$ is the modulus. It is usual to invoke the ergodic hypothesis, so that ensemble averages $\langle\cdot\rangle$ are considered as representing time averages. The problem can be recast as the solution to the following EulerLagrange integral equation:

$$
\iint_{\Omega}\left\langle u^{\prime}(x, y) \otimes u^{\prime *}\left(x^{\prime}, y^{\prime}\right)\right\rangle \psi\left(x^{\prime}, y^{\prime}\right) \mathrm{d} x^{\prime} \mathrm{d} y^{\prime}=\lambda \psi(x, y),
$$

where $\otimes$ is the tensor product, $*$ denotes complex conjugation, and $[x, y] \in \Omega$. The kernel of Eq. (3) is the averaged autocorrelation tensor

$$
R\left(x, x^{\prime} ; y, y^{\prime}\right)=\left\langle u^{\prime}\left(x, y, t_{n}\right) \otimes u^{\prime *}\left(x^{\prime}, y^{\prime}, t_{n}\right)\right\rangle .
$$

The non-negative and self-adjoint properties of $R\left(x, x^{\prime} ; y, y^{\prime}\right)$ ensure that all eigenvalues are real and non-negative, and can therefore be ordered such that $\lambda_{n} \geqslant \lambda_{n+1} \geqslant \cdots \geqslant 0$. In practice, results from the experiments give snapshots of data at a finite number of discrete points, and these data can be placed into matrix form. $R\left(x, x^{\prime} ; y, y^{\prime}\right)$ is thus given by

$$
R\left(x, x^{\prime} ; y, y^{\prime}\right)=\frac{1}{M} \sum_{n=1}^{M} u^{\prime}\left(x, y, t_{n}\right) u^{\prime}\left(x^{\prime}, y^{\prime}, t_{n}\right) .
$$

The snapshot POD method, as first proposed by Sirovich, ${ }^{38}$ is implemented in the present study. The snapshot method makes use of the fact that $u^{\prime}\left(x, y, t_{n}\right)$ and $\psi_{k}(x, y)$ span the same linear space. Thus, the POD eigenmodes can be written as a linear sum of the data vectors

$$
\psi_{k}(x, y)=\sum_{n=1}^{M} \Phi_{n}^{k} u^{\prime}\left(x, y, t_{n}\right), \quad k=1, \ldots, M,
$$

where $\Phi_{n}^{k}$ is the $n$th component of the $k$ th eigenvector. The eigenmodes can then be found by solving the following eigenvalue problem:

$$
C \Phi=\lambda \Phi .
$$

In this way, the eigenvectors of the $N \times N$ matrix $R$ can be found, by computing the $M \times M$ matrix $C$-an attractive method since in the present study $M \ll N$ [where $M=400$ for each test case, with each snapshot containing vector fields of size $\left.N=168\left(N_{x}\right) \times 126\left(N_{y}\right) \simeq 20000\right]$. The reconstruction of any of the original snapshots using an arbitrary number of modes $K$ can be performed with

$$
u\left(x, y, t_{n}\right)=\bar{u}(x, y)+\sum_{k=1}^{K} a_{k}\left(t_{n}\right) \psi_{k}(x, y) .
$$

Important properties are the following orthogonality conditions:

$$
\begin{aligned}
& \left(\psi_{k}(x, y), \psi_{l}^{*}(x, y)\right)=\delta_{k l}, \\
& \left\langle a_{k}\left(t_{n}\right) a_{l}^{*}\left(t_{n}\right)\right\rangle=\lambda_{k} \delta_{k l},
\end{aligned}
$$

where $\delta_{k l}$ is the Kronecker delta. The total energy of the flow is defined as the mean square fluctuating value of velocity, and is given by the sum of the eigenvalues $\lambda_{k}$, each eigenmode being assigned an energy percentage based upon the eigenmode's specific eigenvalue, such that

$$
E_{k}=\lambda_{k} / \sum_{i=1}^{M} \lambda_{i} .
$$

The ensemble size $M$ now becomes important because it is directly linked with the size of the matrix $C$. A previous study on the application of POD to PIV data of compressible planar base flows ${ }^{31}$ has indicated that an acceptable degree of statistical convergence is reached if the ensemble typically contains more than 100 realizations.

\section{RESULTS AND DISCUSSION}

\section{Mean flow organization}

Mean velocity streamlines are displayed with velocity vectors and vertical velocity contours in Figs. 2(a)-2(c). Vectors are undersampled in the streamwise direction (showing 1 in 20 in order to better visualize the important flow features). The outer flow can be seen to pass through a series of expansion waves, which emanate from the shoulder of the base. Here, the flow accelerates isentropically to a maximum velocity magnitude at Mach 1.46, 1.78, and 2.27 of approximately $1.19,1.18$, and $1.17 U_{\infty}$, respectively. The system of expansion waves does not focus exactly at the shoulder of the base due to the finite thickness of the boundary layer. Since the local expansion strength decreases with distance from the shoulder, fluid near to the shoulder experiences a more sudden expansion than fluid farther away. The measurement data are therefore compromised in the near shoulder region due to the large flow gradients present, which cause the flow to rapidly accelerate. This introduces a difference between the measured and theoretical velocity pattern due to particle lag. A previous planar base flow study using $\mathrm{PIV}^{6}$ has determined that the associated error falls to below $1 \%$ for $x / h>0.25$ from the shoulder of the base. Another common concern in the measurement of these types of sepa- 

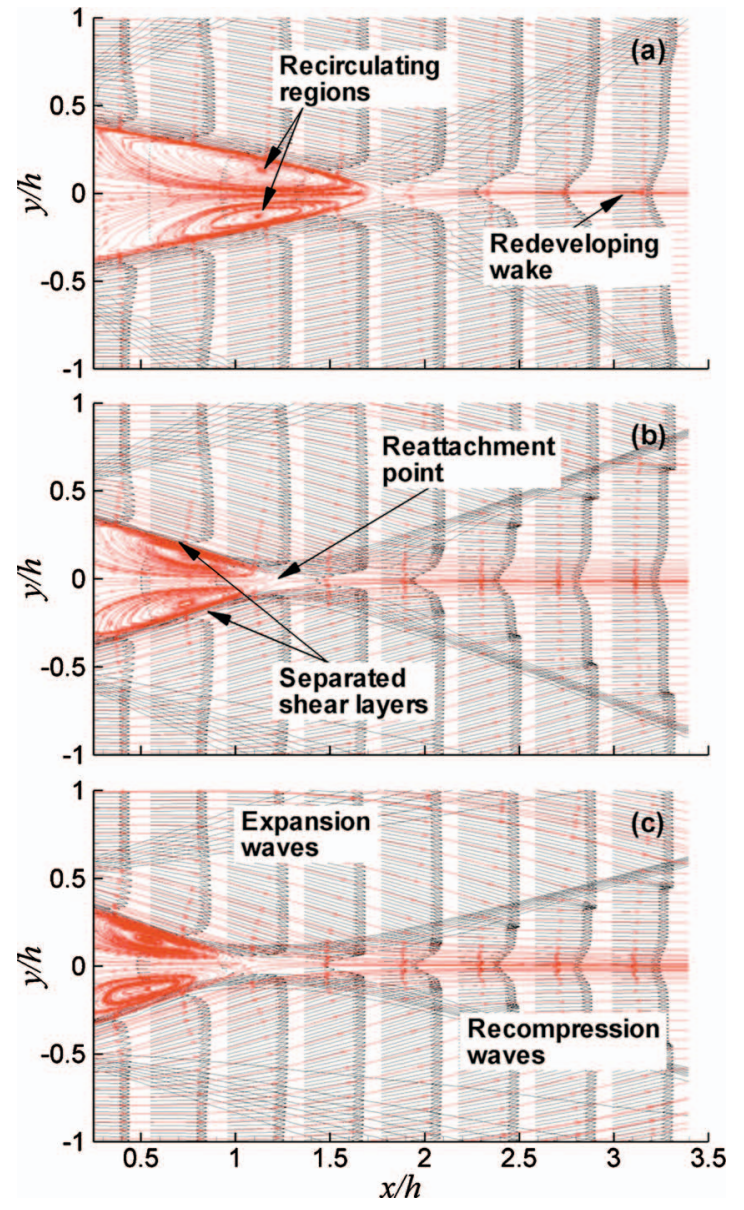

FIG. 2. (Color) Mean flow topology: (a) Mach 1.46, (b) Mach 1.78, (c) Mach 2.27. Mean velocity streamlines are shown with vertical velocity contours. Velocity vectors show 1 in 20.

rated flows is the downstream interference from the initial bow shock wave, as it reflects off the tunnel walls and back toward the redeveloping wake. The streamwise location of the impingement of the reflected bow shock wave back onto the redeveloping wake $x_{\text {reflect }}$ was estimated using isentropic flow theory. It was determined to be $x_{\text {reflect }} / h>7$ at Mach 1.78 , as measured from the base edge. This distance increases with increasing Mach number. Note that the flow approaching the model in the Mach 1.46 case was subsonic, and thus there is no bow shock wave.

Mean flow streamlines verify the symmetry of the mean flow topology. The shear layers undergo a recompression and realignment process, eventually reattaching at a distance $x_{r}$ along the centerline of the wake. For Mach 1.46, 1.78, and 2.27 , reattachment occurs at $x_{r} / h=1.76,1.25$, and 1.1 , respectively, consistent with other results. ${ }^{6}$ Fluid that has insufficient momentum to overcome the pressure gradient at reattachment is directed back toward the base, leading to the formation of two well-defined recirculating flow regions. The recirculating fluid accelerates from the reattachment point to a maximum reversed-flow velocity of $0.19,0.27$, and $0.28 U_{\infty}$, at Mach 1.46, 1.78, and 2.27, respectively. It appears that a similarity relationship exists for the centerline velocity distribution, since the present distributions were found to change little over the Mach number range considered. Spu-

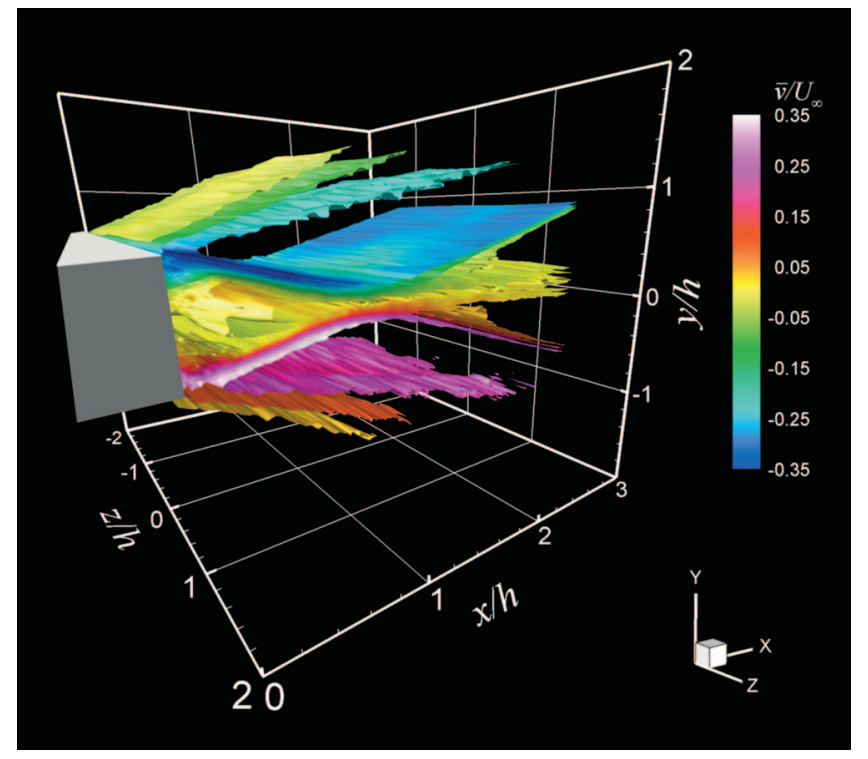

FIG. 3. (Color) Spanwise survey of the near wake region. Isosurfaces of mean streamwise velocity are shown flooded with mean vertical velocity.

rious streamlines close to the base wall could be observed in all test cases (typically for $x / h<0.1$ ). These were due to laser light reflections from the base.

The separated free shear layers appear as densely spaced velocity contours, which approach each other in the reattachment region, spreading slightly due to the turbulent mixing. Downstream of reattachment, a mutual interaction takes place, and the spreading of the velocity profiles becomes more pronounced. Flow recompression is marked by the appearance of compression waves, which emanate from the reattachment region. These coalesce to form the oblique shock waves. A recovery process of the wake deficit occurs farther downstream, although the complete recovery process cannot be observed within the present measurement domain. Overall, it is clear that the spatial extent of the near wake decreases with increasing Mach number, with the expansion and oblique shock waves becoming more inclined toward the wake axis.

\section{Two-dimensionality of the mean flowfield}

As noted by Amatucci et al., ${ }^{5}$ nominally twodimensional flowfields that are characterized by a large-scale separated flow region may exhibit spanwise cellular nonuniformity. To examine this effect in the present study, a multiplanar assessment of the near wake was carried out at Mach 1.78 , within the range $-2.0 \leqslant z / \delta \leqslant 2.0$, in increments of $0.5 \delta$ (i.e., nine planes). A total of 60 image pairs were obtained at each spanwise location. Figure 3 shows isosurfaces of the mean streamwise velocity, flooded with contours of vertical velocity. From these results, it can be seen that the mean velocity remains quite uniform over the spanwise region considered. There appears to be no significant influence of the sidewall boundary layers, nor evidence of wind tunnel irregularities. However, the measured flow properties show an appreciable deviation from the centerline values at distances from the centerline greater than $15 \%$ of the test sec- 

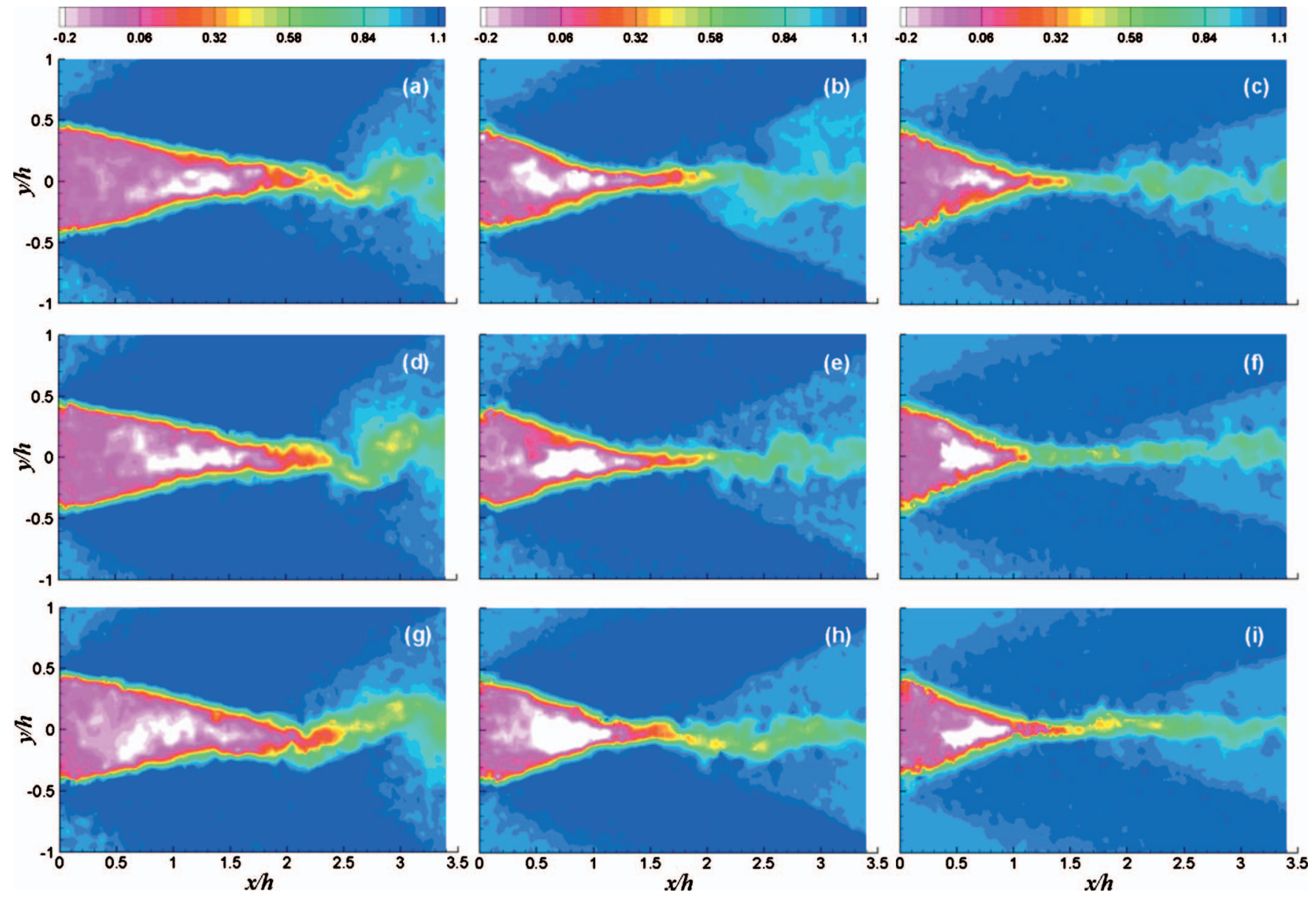

FIG. 4. (Color) Instantaneous streamwise velocity distribution $u / U_{\infty}$ : (left) Mach 1.46, (center) Mach 1.78, (right) Mach 2.27.

tion width. This behavior is ascribed to the lower measurement confidence level, due to the increasingly intermittent nature of the incoming seeded streamtube.

\section{Instantaneous flow organization}

In order to first give a relatively general description of the organized global dynamics, several fields of the instantaneous streamwise velocity component are illustrated in Figs. 4(a)-4(i). These fields typify the dynamical events that are observed to take place. The time that elapses between consecutive recordings (5 Hz framing rate) is significantly greater than any characteristic flow time scale, which leads to uncorrelated velocity snapshots. The instantaneous results reveal that the structure of the near wake region varies considerably in time. In general, it can be seen that the more asymmetric the near wake region becomes, the greater the movement of the reattachment region. This behavior is similar to what has been observed in POD flow analyses of an annular jet. ${ }^{39}$ Interestingly, while a unique reattachment point can be easily determined from the mean velocity field, the flow often stagnates at several locations instantaneously.

The structure of the near wake region consists of a variety of organized dynamics, or dynamical features, which extend over streamwise length scales that are considered large in comparison to the length scales associated with the instabilities that cause them. The underlying global dynamics that arise from these instabilities can therefore be quite complicated. Before discussing them in more detail, let us first establish the concept of a global mode, as referring to an organized global dynamical behavior of the near wake region. Thus far, we have been rather casual in our description of the organized global dynamics, and we must now return to this notion. A global mode will be describable in terms of a global eigenmode, which can essentially be viewed as being a perturbation of the mean flow. Each eigenmode is constructed out of the spatial organization of the fluctuating part of the flowfield in the most efficient manner. That is, an optimal set of basis functions are generated that represent the "energy" of the data, defined by our user-selected norm, which is the mean square fluctuating value of the velocity. This basis is optimal in the sense that a finite number of these modes represent more of the energy than any other set of orthogonal modes. In reality, the complex behavior of the near wake region can be shown to consist of many global eigenmodes, which can in fact be used together in an appropriate combination to reconstruct an individual dynamical state, or velocity field obtained from the experiments. In what follows, we restrict our attention to discussing only the most predominant global modes observed in the experiments, which correspond to the most dynamically significant organized motions. (It will be shown that the most predominant global modes are related to the most energetic eigen- 
modes.) With this terminology at hand, we can see by inspection that each of the considered flows exhibit a different instantaneous flow organization, whose overall general behavior can be characterized by a predominant global mode.

A significant vertical motion of the near wake is observed at Mach 1.46, and we qualitatively characterize the global mode in this case as being a sinuous or flapping motion, similar to the well-known von Kármán vortex street commonly observed in many incompressible wakes. This flapping motion has an amplitude that is comparable to the mean wake width. The unsteady motion is particularly evident within the redeveloping wake region, where the vortex shedding process takes place. Bourdon and Dutton ${ }^{40}$ have examined shear layer flapping in a supersonic axisymmetric base flow. They found that the flapping generally increases with downstream development, reaching root-mean-square displacements of up to $40 \%$ of the local thickness. They further noted that within the wake redevelopment region, the flapping becomes particularly significant when compared to the base radius. These trends are consistent with the present observations made at Mach 1.46. (The reader can confirm this by looking ahead to the turbulence statistics that will be presented, bearing in mind that organized motions have a significant effect on the overall turbulence structure.) Interestingly, it was also shown in the work by Bourdon and Dutton that planar shear layer results were typically larger than in their axisymmetric base flow. This is also substantiated by the present results, which show a flapping motion that is of the order of the redeveloping wake width. It has been suggested that the geometrical constraints imposed by the axisymmetric shear layer dampens the large-scale motions.

The present results show that as the Mach number increases, the amplitude of the flapping motion decreases, along with the large vertical velocity fluctuations typically associated with the vortex shedding process. Indeed, it is well known that vortex shedding is weak in supersonic flows. ${ }^{32,33}$ Upon closer inspection, it can be seen that not only does this flapping motion decrease with increasing Mach number, but the motion of the reattachment region develops a more streamwise orientation. (This observation will be further evidenced in the POD analysis to be presented later, and is substantiated by the turbulence statistics that follow.) We may therefore conceptualize the predominant global mode at Mach 2.27 as being characterized by a pulsating motion aligned with the wake axis. Observe how the reattachment location moves a streamwise distance that is typically of the order of the redeveloping wake width. Scarano and van Oudheusden, ${ }^{6}$ using a low-pass filtered version of the velocity field, have determined the spatial occurrence of the instantaneous reattachment location for the same planar base flow at Mach 2. They found that the spatial fluctuations were indeed larger in the streamwise direction than in the vertical direction, suggesting that the overall global dynamics were dominated by a pulsating motion. Interestingly, there is evidence suggested by Clemens and Mungal ${ }^{41}$ that the organization of the large-scale turbulent structures develop a more streamwise orientation with increasing compressibility.
One particularly interesting fluid dynamic feature of the present results is that the predominant global modes appear to influence the motion of the recompression shock waves, which emanate from the reattachment region. Inspection of the individual instantaneous velocity vector fields reveals that the recompression shock waves generally move upstream when wake streamlines in the reattachment region become temporarily concave, and move downstream when wake streamlines become temporarily convex. This observation is more apparent at Mach 1.46, as would be expected. [Figure 4(d) is a typical example.] This behavior has in fact also been noted by Kastengren et ll $^{42}$ in their recent reattachment shock wave visualization studies using Mie scattering. Furthermore, the recompression shock waves can be seen to generally move downstream when the near wake region becomes temporarily larger, and move upstream when the near wake region becomes temporarily smaller. This observation is more apparent at Mach 2.27, and has also been documented in earlier work by the present authors. ${ }^{31}$ Another interesting feature is the occurrence of an expansion fan where the redeveloping wake becomes locally convex [Figs. 4(d) and 4(g) are typical examples illustrating this]. Here, the expansion fan can be seen to influence the overall compression that takes place in the outer flow. Although particularly evident at Mach 1.46, this observation can also be made at Mach 1.78 and 2.27. The foregoing results highlight some important observations regarding the instantaneous near wake behavior, which will be used in our subsequent discussion. It is important to emphasize that these observations are supported by experience with hundreds of velocity fields, as well as those obtained in other PIV compressible planar base flow studies, ${ }^{6,31}$ which have considered additional intermediate Mach numbers not considered here.

\section{Turbulence statistics}

Turbulence statistics are now presented because we wish to examine the effect of the organized global dynamics on the distributed nature of the turbulence properties. Distributions of the streamwise turbulence intensity $\left\langle u^{\prime}\right\rangle$ for the test cases considered are shown in Figs. 5(a)-5(c). Relatively moderate levels can be observed within the separated shear layers. The location of the peak $\left\langle u^{\prime}\right\rangle$ in this region appears to coincide with the inflection point of the mean velocity profile, which is centered between the edges of the shear layer, consistent with observations that have been made in planar compressible base flows. ${ }^{6}$ Detailed LDV studies of axisymmetric separating shear layers ${ }^{8}$ have shown that the effect of the separation process through the rapid expansion at the shoulder is in fact to magnify $\left\langle u^{\prime}\right\rangle$ along the inner edge of the shear layer. Further work is required to clarify the similarities and differences between planar and axisymmetric shear layers.

The present values of $\left\langle u^{\prime}\right\rangle$ throughout the near wake region compare favorably with the results of other supersonic planar base flows, ${ }^{5,6}$ as well as backward facing steps ${ }^{43}$ and axisymmetric configurations, ${ }^{8}$ which reach levels of up to $25 \%$ of $U_{\infty}$ (see also Fig. 8). As the flow enters the reattachment region, a significant broadening of $\left\langle u^{\prime}\right\rangle$ occurs due to 

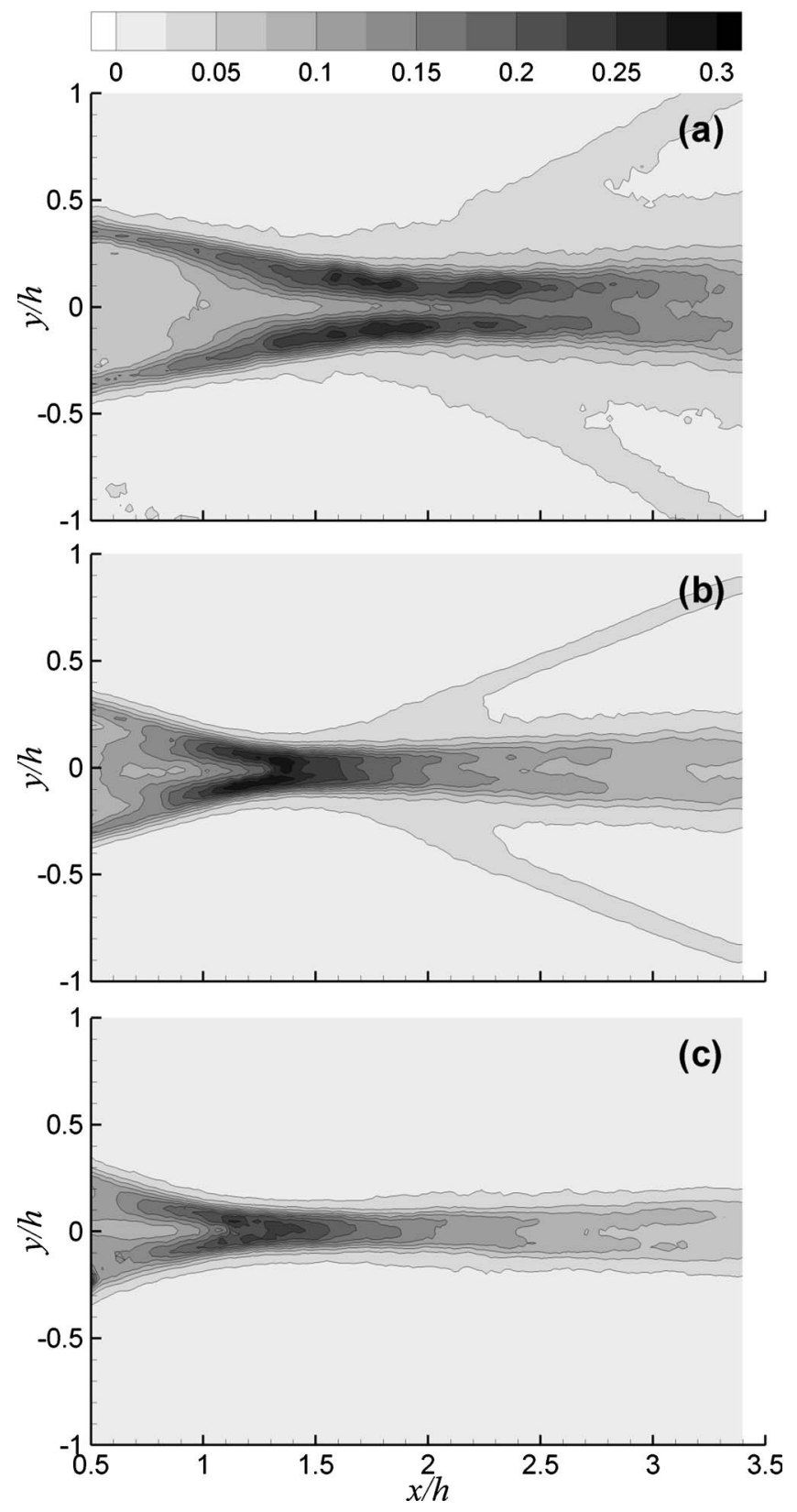

FIG. 5. Streamwise turbulence intensity distribution $\left\langle u^{\prime}\right\rangle / U_{\infty}$ : (a) Mach 1.46, (b) Mach 1.78, (c) Mach 2.27.

the large-scale interaction, as well as the increase in turbulence activity along the wake axis, as a result of the mutual shear layer interaction. Here, global maximum levels of the $\left\langle u^{\prime}\right\rangle$ component are obtained. The two shear layers of opposite spanwise vorticity begin to merge, and the velocity deficit thereafter begins to recover. Although two distinct streamwise turbulence intensity peaks indicating the approaching shear layers are initially present, their merging appears to be rather different at Mach 1.46 than at Mach 1.78 and 2.27. While in the latter two cases there is a strong merging, resulting in a single turbulence peak within the reattachment region, in the former case there is a somewhat weaker merging farther downstream. Clearly, there is some question of how the global mode dynamics affect the structure of the turbulence properties, but the present evidence suggests that
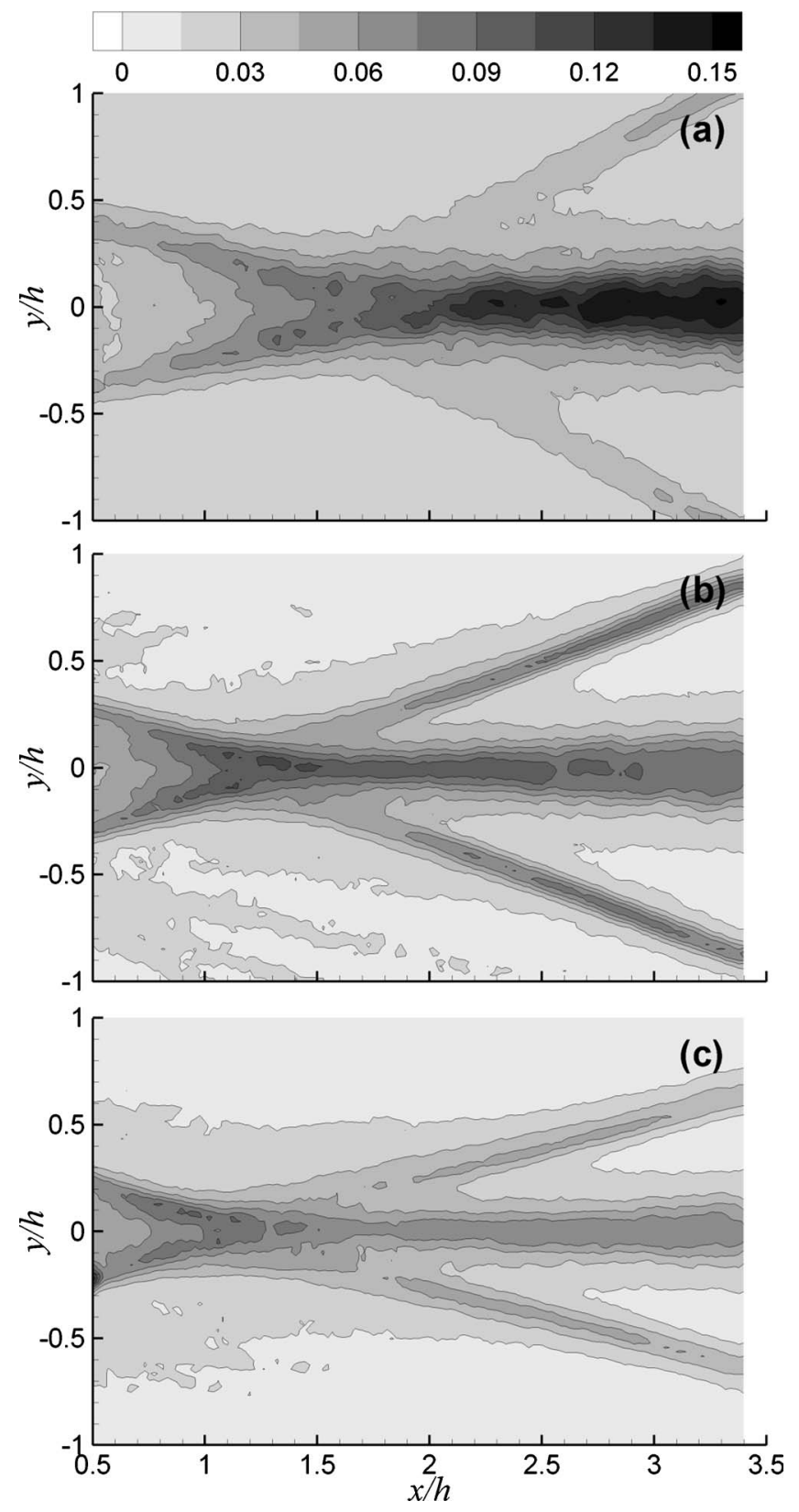

FIG. 6. Vertical turbulence intensity distribution $\left\langle v^{\prime}\right\rangle / U_{\infty}$ : (a) Mach 1.46, (b) Mach 1.78, (c) Mach 2.27.

there is an important role being played by the predominant global mode in determining the distributed nature of the turbulence properties within the near wake region.

The corresponding vertical turbulence intensity $\left\langle v^{\prime}\right\rangle$ distributions are shown in Figs. 6(a)-6(c). The scale is now twice as sensitive as $\left\langle u^{\prime}\right\rangle$. The results show initially moderate levels within the separated shear layers, with significantly elevated levels observed within the reattachment and wake redevelopment regions. At Mach 1.46, $\left\langle v^{\prime}\right\rangle$ appears to spread rather broadly over the vertical height of the interaction downstream within the redeveloping wake. In contrast, at Mach 1.78 and 2.27, there is an appreciable decay of $\left\langle v^{\prime}\right\rangle$ downstream. This is consistent with the idea that the flapping mode decreases with increasing Mach number, being replaced with a more streamwise-oriented unsteady wake mo- 

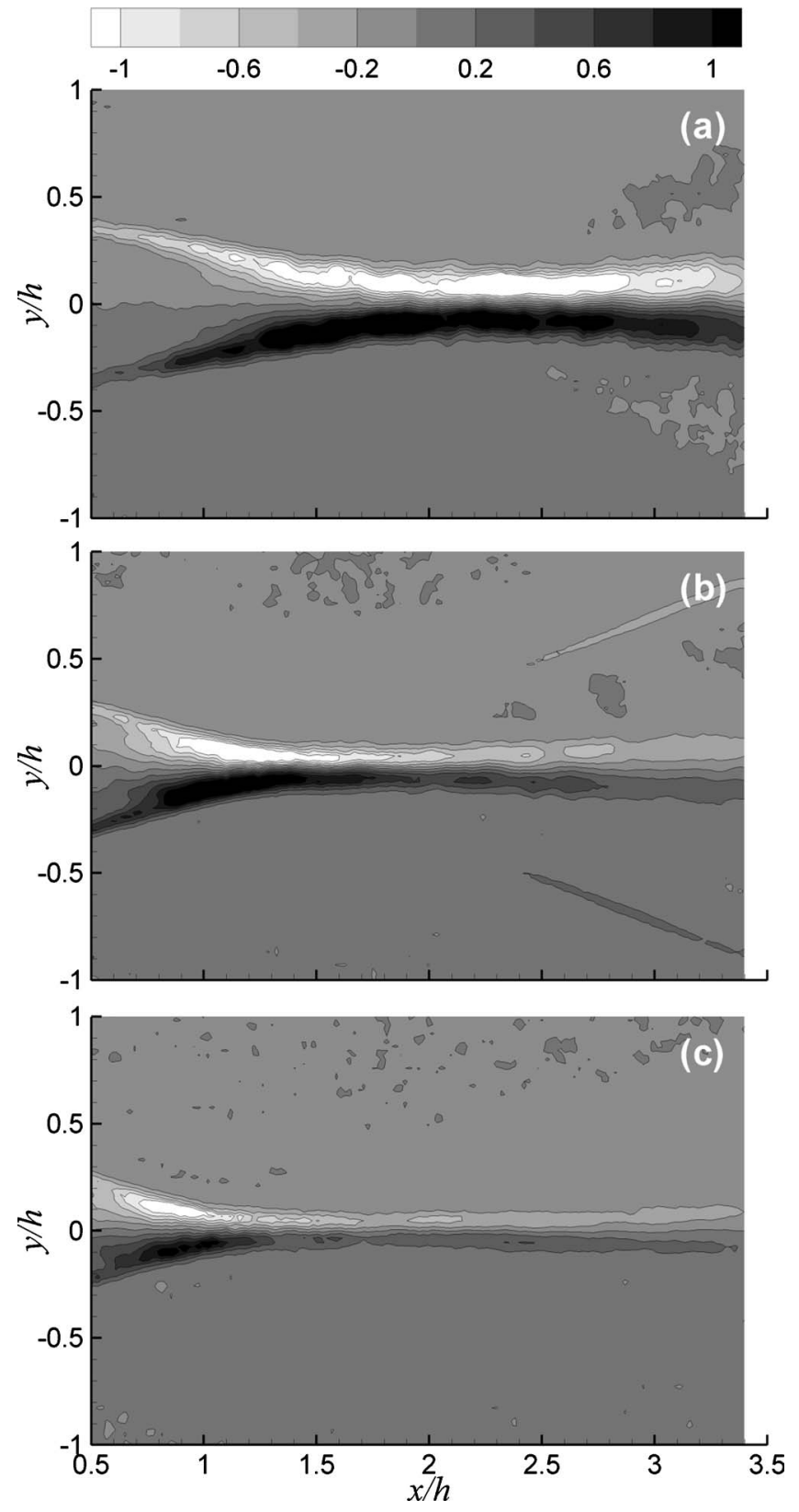

FIG. 7. Kinematic Reynolds shear stress distribution $-\overline{u^{\prime} v^{\prime}} / U_{\infty}^{2} \times 10^{2}$ : (a) Mach 1.46, (b) Mach 1.78, (c) Mach 2.27.

tion. The turbulent mixing processes therefore remain confined to a much narrower redeveloping wake width with increasing compressibility, as a result of the diminished vortex street. This again highlights the important impact of the predominant global modes on the turbulence properties. At some point downstream of the reattachment region, we can anticipate that the change in flow regime from a shear layer into a wake will eventually lead to significantly reduced turbulence levels, since the wake does not contain the relatively large mean shear rates as those present in the approaching shear layers. Note the higher level of fluctuations associated with the oblique shock waves, which is typically encountered in these experimental conditions due their unsteady motion.

The kinematic Reynolds shear stress distributions $\overline{u^{\prime} v^{\prime}} / U_{\infty}^{2}$ are shown in Figs. 7(a)-7(c). Such measurements

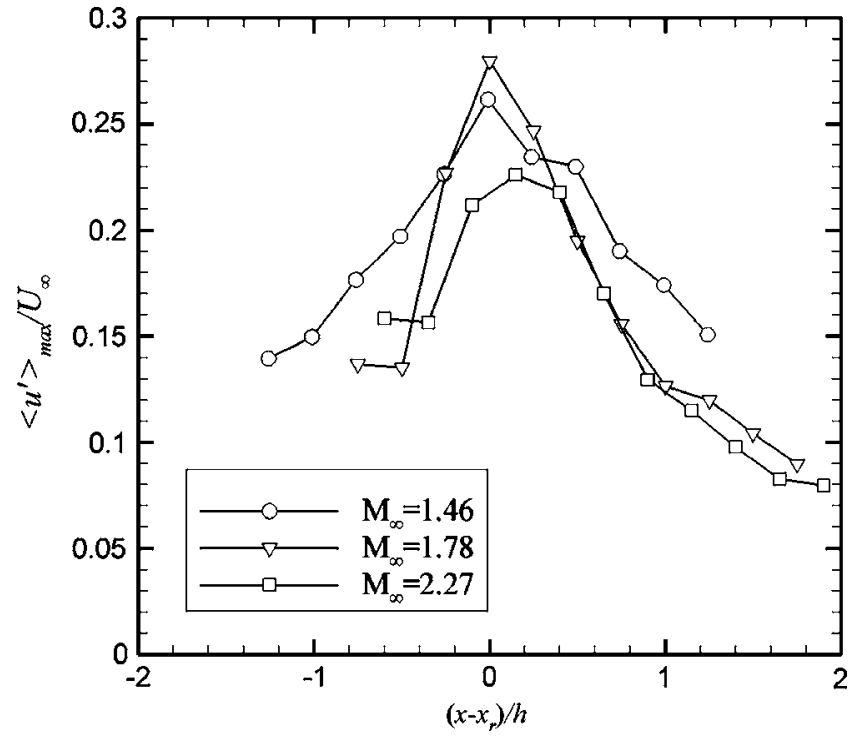

FIG. 8. Evolution of the maximum streamwise turbulence intensity $\left\langle u^{\prime}\right\rangle_{\max } / U_{\infty}$

are principally carried out to aid the modeling of turbulent effects by computational methods. Initially moderate levels are present within the separated shear layers, similar to what has been observed in the turbulence intensity distributions, except along the centerline, where the kinematic Reynolds shear stress must vanish by symmetry. These levels increase significantly as the reattachment region is approached, and two well-defined, broad regions of kinematic Reynolds shear stress persist downstream into the redeveloping wake. Note that within the shear layers, the magnitude of the turbulence properties increases with streamwise development, indicating that a mixing and entrainment process takes place along the shear layer boundaries.

The maximum contributions from each component are important to the improved understanding of the evolving nature of the near wake turbulence structure. The evolution of the local maximum levels of $\left\langle u^{\prime}\right\rangle$ and $\left\langle v^{\prime}\right\rangle$ are shown in Figs. 8 and 9 , respectively, along the normalized coordinate $(x$ $\left.-x_{r}\right) / h$. Both components increase from their relatively moderate levels within the shear layers, to reach global maximum levels within the reattachment region. To be precise, $\left\langle u^{\prime}\right\rangle$ reaches its global maximum in the vicinity of the mean reattachment point, and does not appear to exhibit any systematic trend with compressibility, within the Mach number range considered. Farther downstream, a rapid decrease of $\left\langle u^{\prime}\right\rangle$ occurs within the redeveloping wake region. This can be contrasted with the behavior of the $\left\langle v^{\prime}\right\rangle$ component. Here, we see that a systematic movement of the global maximum of $\left\langle v^{\prime}\right\rangle$ occurs with increasing compressibility; namely, a movement from the redeveloping wake, upstream toward the mean reattachment point. This behavior is attributed to the decreasing action of the alternately shed vortices, which lead to a reduced alternating flow entrainment pattern of vertical velocity. It is interesting to note that the $\left\langle v^{\prime}\right\rangle$ component typically undergoes a slower recovery downstream of its global maximum in comparison to $\left\langle u^{\prime}\right\rangle$.

To further explain these trends, it is necessary to better 


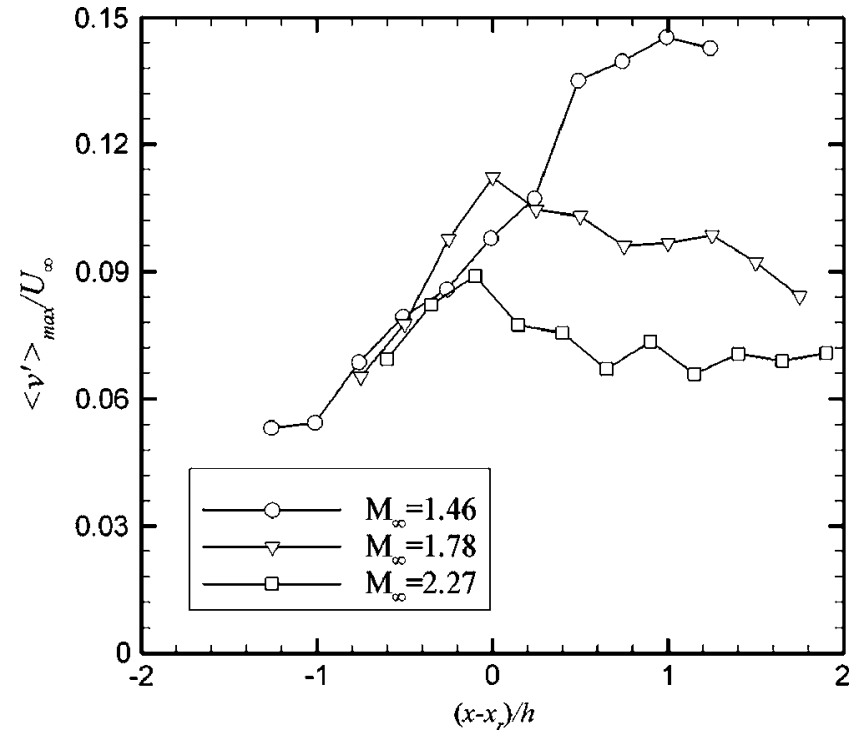

FIG. 9. Evolution of the maximum vertical turbulence intensity $\left\langle v^{\prime}\right\rangle_{\max } / U_{\infty}$.

understand the physical processes taking place. The transfer of kinetic energy from the mean flow is essentially to $\left\langle u^{\prime}\right\rangle$ by normal advection across the mean velocity gradient. Since $\left\langle u^{\prime}\right\rangle$ is generally larger than both $\left\langle v^{\prime}\right\rangle$ and the spanwise component $\left\langle w^{\prime}\right\rangle$, energy is redistributed to these latter two terms. Therefore, similar to what occurs in axisymmetric base flows, the transfer of energy from the mean flow to the turbulence field takes place via the classical production mechanisms to the streamwise component $\left\langle u^{\prime}\right\rangle$, whereas the vertical and spanwise components acquire energy through the more passive pressure-strain correlation terms and turbulent diffusion. ${ }^{44}$ This results in significant turbulence anisotropy within the near wake region and explains the very different turbulence amplifications of the turbulence intensities.

Direct numerical simulations (DNS) performed by Friedrich et $a .^{45}$ have demonstrated that the absolute pressure-strain correlation terms are typically smaller in compressible flows than in corresponding incompressible flows. One might therefore intuitively expect that the energy transfer process due to the pressure-strain correlation terms will diminish with increasing compressibility, thereby suppressing the redistribution of streamwise turbulence energy to the other components. This may explain why the maximum streamwise intensity remains somewhat unaffected, if not decreased with increasing compressibility, whereas the vertical turbulence intensity decreases significantly, and recovers more slowly than the streamwise component. Interestingly, the streamwise component remains systematically higher than the vertical component, despite its rapid recovery downstream of the reattachment region. It would therefore appear that the relatively large $\left\langle u^{\prime}\right\rangle$ production does not necessarily balance the tendency toward isotropy, an observation that has been attributed to the insufficient streamwise extent of the interaction process. ${ }^{46}$

Maximum levels of the kinematic Reynolds shear stress throughout the near wake region are shown in Fig. 10. The kinematic Reynolds shear stress increases rapidly upon approaching the mean reattachment point, reaching global

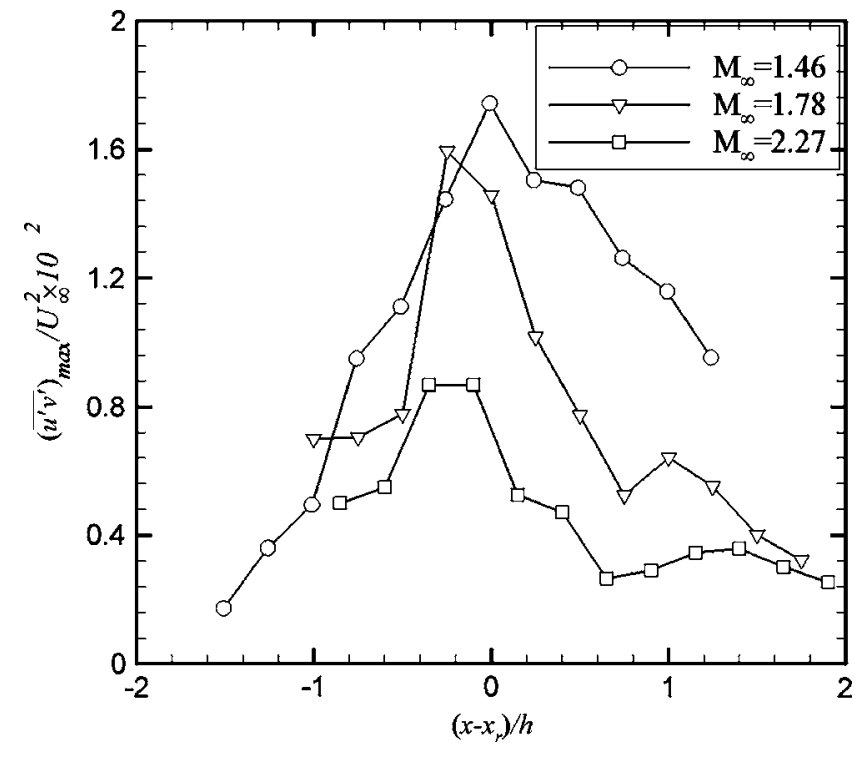

FIG. 10. Evolution of the maximum kinematic Reynolds shear stress.

maximum levels of approximately $1.8 \%, 1.6 \%$, and $0.8 \%$ at Mach 1.46, 1.78, and 2.27, respectively. Clearly, there is a systematic decrease in the maximum kinematic shear stress with increasing compressibility. These levels are consistent with the study made by Samimy et $a l^{47}$ on a twodimensional, reattaching shear layer at Mach 2.46, which shows a maximum shear stress (without the density term) leveling off at approximately $0.5 \%$ in the reattachment region. Also, in a study of the interaction between two compressible, turbulent shear layers at Mach 1.50 and 2.07, Samimy and $\mathrm{Addy}^{7}$ report a maximum kinematic Reynolds shear stress of approximately $1.5 \%$ at Mach 1.50 , and $1.1 \%$ at Mach 2.07, again consistent with the present results. The increase in kinematic Reynolds shear stress is the result of the overall destabilizing effects of streamline curvature and bulk compression. ${ }^{8}$ The kinematic Reynolds shear stress profiles tend to peak sharply in the vicinity of the mean reattachment point, followed by a rapid decay farther downstream, very similar to what has been observed by Amatucci et $a .^{5}$ in their two-dimensional supersonic base flow investigation. This is the result of the shear layer alignment process and the progressive decrease in mean shear rates as the shear layers develop into a wake flow. This behavior can be contrasted with what is known to occur in compressible axisymmetric base flows. Here, the turbulent Reynolds stresses have been shown to actually decrease throughout the recompression and reattachment regions. ${ }^{8}$ This difference in behavior between planar and axisymmetric base flows has been hypothesized to be the result of the overwhelming effects of lateral streamline curvature, which provides a stabilizing influence that is not present in the planar case. A similar qualitative trend also occurs in subsonic, solid wall cases. ${ }^{48} \mathrm{Al}-$ though the precise cause(s) for this behavior are not known, it seems that compressibility effects are more predominant in supersonic flow reattachment, ${ }^{47}$ at least in the twodimensional, supersonic compliant case. ${ }^{8}$

It is now clear that differences in the underlying unsteady flow organization (as well as the type of configura- 
tion) play an important role in determining the distributed nature of the turbulence properties within the near wake region. As well as the differences observed in their distributed nature, there is also a disparity between the overall turbulence levels with increasing compressibility. Generally, the local maximum turbulence intensities and kinematic Reynolds shear stress can be seen to decrease with increasing compressibility, within the Mach number range considered. This behavior may be the result of an increasingly weaker separation and expansion process with increasing Mach number, as suggested by Samimy and $\operatorname{Addy}^{7}$ (and somewhat substantiated by the decrease in the maximum velocity ratio attained within the expansion region reported in the present study), or it may be the consequence of a higher convective Mach number, as suggested by Papamoschou and Roshko. ${ }^{49}$ The correlation coefficient $R_{u v}=\overline{u^{\prime} v^{\prime}} /\left\langle u^{\prime}\right\rangle\left\langle v^{\prime}\right\rangle$, however, appears to remain unchanged from its incompressible value, being typically within the range -0.5 to -0.6 for the test cases considered.

\section{POD analysis}

Having conceptually outlined some important observations regarding the underlying unsteady flow organizations of the flows considered, we now wish to construct a loworder representation of their complex dynamical behavior. A statistical evaluation of the velocity fluctuations based upon the PIV data is carried out using the POD snapshot method described above. Physically, each eigenmode can be considered as capturing an independent predominant dynamical characteristic of the flow, which may not be intuitively revealed by the instantaneous realizations. Each eigenmode therefore represents a global mode. Although the interpretation of eigenmodes as representing physical flow phenomena has long been a source of debate, a general consensus is that it relies chiefly upon the energy convergence. As a motivating prelude, the eigenmode energy and cumulative eigenmode energy distributions for the test cases considered are presented in Figs. 11 and 12, respectively.

The present eigenmode energy distributions generally reflect a poor energy convergence when compared to other POD analyses, including wake flows, ${ }^{50-52}$ where a larger amount of energy is typically captured in a relatively smaller number of eigenmodes. This discrepancy is attributed to the comparably high Reynolds numbers in the present experiments, which lead to energy being distributed among a larger number of modes, since many flow scales are captured in an instantaneous flowfield. Furthermore, the presence of random noise and occasional poor data quality, naturally present in experimental data, also contribute to flattening the eigenspectra distribution, by transferring energy toward the higher-order eigenmodes.

The results show that a systematically lower energy convergence occurs with increasing Mach number. Almost 50\% of the total energy is captured by the first 10 eigenmodes at Mach 1.46, compared with 39\% and 33\% at Mach 1.78 and 2.27 , respectively. This trend is ascribed to the fact that the motion of the large-scale organized dynamics becomes increasingly deterministic with decreasing Mach number, in

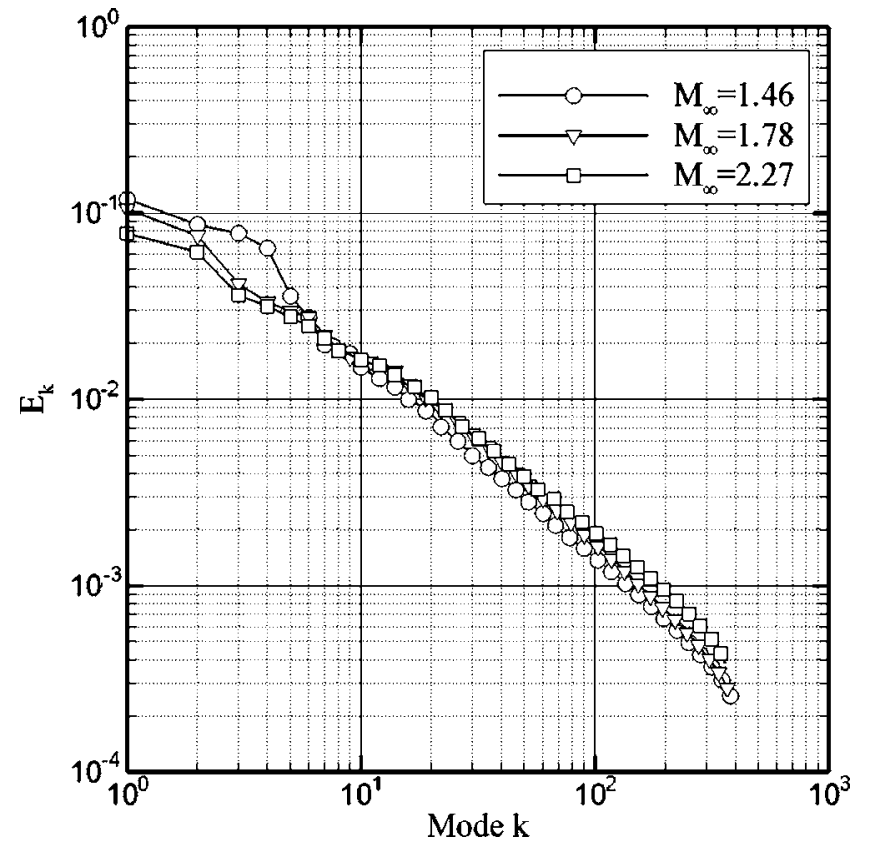

FIG. 11. Eigenmode energy distribution.

the sense that a well-organized, large-scale von Kármán type wake becomes predominant, such that the energy associated with this motion is captured increasingly more effectively by the lower-order eigenmodes. The present eigenmodes are considered capable of providing a good basis for a general discussion about the nature of the flows, since it will be shown that conclusions can be drawn that are consistent with the observations made in the PIV instantaneous realizations.

A selection of eigenmodes depicting the normalized streamwise velocity component are shown in Figs. 13(a)-13(1). These eigenmodes are considered to be those that best represent the unsteady nature of the flows. Interme-

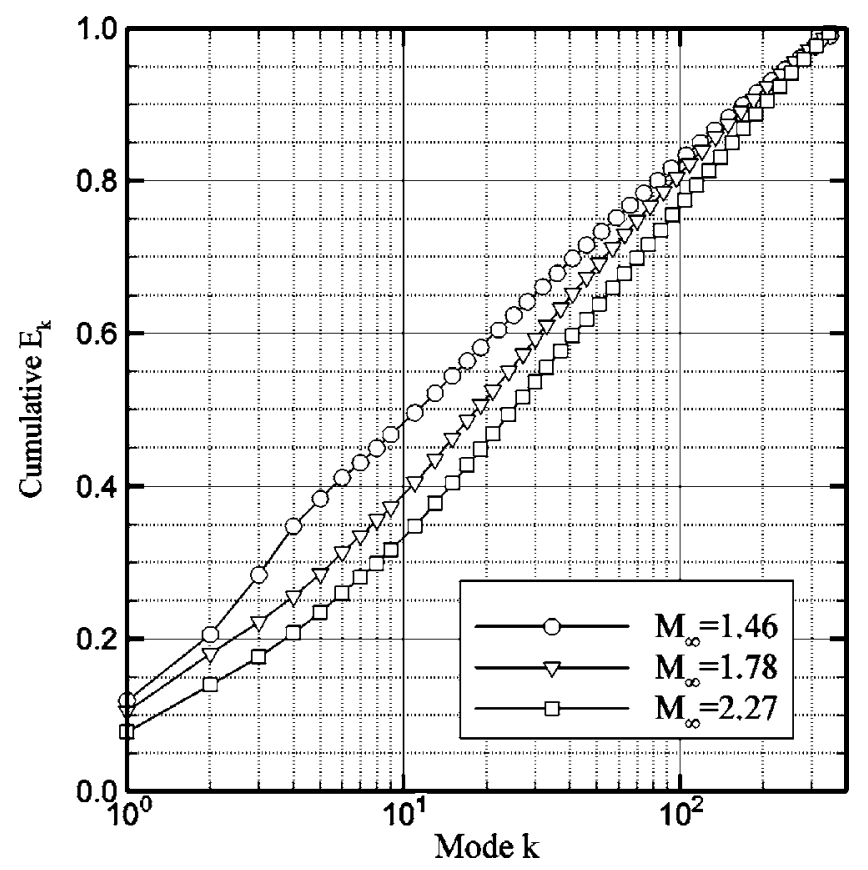

FIG. 12. Cumulative eigenmode energy distribution. 


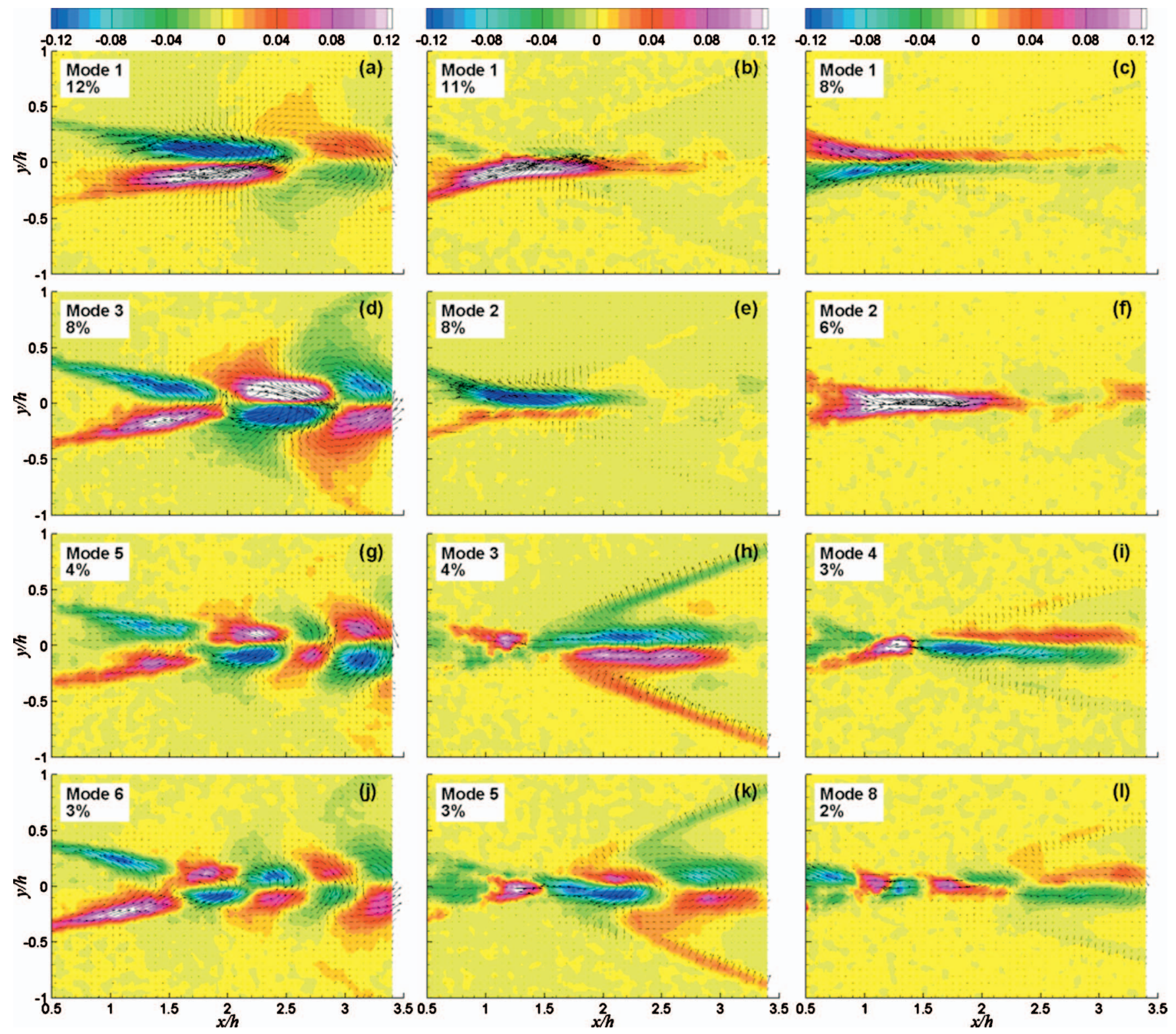

FIG. 13. (Color) POD eigenmodes: streamwise velocity component $u^{\prime} / U_{\infty}$ : (left) Mach 1.46, (center) Mach 1.78, (right) Mach 2.27. Mode number and energy content shown (inset). Note that $2 u^{\prime} / U_{\infty}$ is shown for modes $\geqslant 3$.

diate eigenmodes typically show the same fundamental features and have therefore been omitted. The eigenmodes are sorted by decreasing fractional energy and also display velocity vectors, undersampled in order to improve the clarity of the results. Also, note the offset in the abscissa, since data for $x / h<0.5$ were not considered in the POD analysis. This was due to the spurious velocity fluctuations that occurred, as a result of the strong laser light reflections in the proximity of the base. These spurious velocity fluctuations were found to interfere with the eigenmode constructions.

The low-order eigenmodes represent the most energetic modes, and therefore best represent the fluctuating part of the flowfield. The first eigenmode of the Mach 1.46, 1.78, and 2.27 cases contain approximately $12 \%, 11 \%$, and $8 \%$ of the total kinetic energy, respectively. Generally, within these first eigenmodes, the largest streamwise velocity fluctuations typically occur within the reattachment region, which is known to be the region of highest turbulent activity in compressible base flows. ${ }^{53}$ It is therefore expected that fluid motions in this region will likely contribute the most toward the total energy of the flow, since it is defined as the mean square fluctuating value of the velocity. More specifically, the eigenmodes at Mach 1.46 display distinct regions of relatively large velocity fluctuations of alternating sign, indicative of the train of quasistreamwise vortices associated with a convected vortex street. ${ }^{54}$ Here, pairing occurs between several of these eigenmodes (e.g., eigenmodes 5 and 6). This pairing can be explained by the fact that the mechanism of the sinuous mode can be represented by a traveling wave composed of eigenmodes in phase quadrature. When one eigenmode in the pair is at a maximum, the other is at a minimum stage. This relation reverses itself at the phase shift between them, with an energy exchange taking place that thereby propagates the flow pattern. This can be contrasted with what is 
observed to occur in the Mach 2.27 case. Here, streamwiseelongated regions of velocity fluctuations can be seen, which are typical of a pulsating motion aligned with the wake axis. ${ }^{31}$ No significant pairing can be observed between the eigenmodes in this case. This is consistent with the idea that the predominant global mode can be characterized by a pulsating motion, represented by a standing wave. Previous planar base flow experiments performed by Kemmoun ${ }^{55}$ at Mach 1.95, using hot-wire anemometry in the same facility, have also found evidence that supports the existence of a predominant pulsating motion. A peak frequency of $7.5 \mathrm{kHz}$ in the region between the expansion fan and free shear layer has been reported. This corresponds to a Strouhal number of 0.3 when based upon the base height $h$ and freestream velocity $U_{\infty}$, which is not dissimilar to the Strouhal numbers obtained in studies of flat plate supersonic wakes. ${ }^{32,33}$ Interestingly, the first two eigenmodes of the Mach 1.78 case appear to form a pair, symmetrically arranged with the wake axis. These eigenmodes can be seen to exhibit both the flapping and pulsating motion observed in the Mach 1.46 and 2.27 cases, respectively, tentatively suggesting that the overall global dynamics may be a combination of these two predominant global modes. This is not immediately obvious from the instantaneous velocity realizations.

Higher-order eigenmodes in all the test cases typically show coherent flow features becoming progressively smaller in spatial extent, as they contain an increasingly smaller fraction of the total kinetic energy. Regions of velocity fluctuations that alternate in sign often appear, symmetrically arranged with the wake axis. This behavior is particularly evident at Mach 1.46, and occurs in the separated shear layers, reattachment, and redeveloping wake regions. Maximum streamwise velocity fluctuations can still, however, be observed in the reattachment region. It is now undisputable that, with increasing compressibility, the streamwise velocity fluctuations become increasingly elongated and aligned with the wake axis. Interestingly, in all eigenmodes considered, very little flow activity is observed in the recirculating flow region. This is consistent with the idea that while turbulence within this region is regarded as being typically high, there is relatively little correlative behavior induced by the smallscale velocity fluctuations.

The corresponding eigenmodes of the normalized vertical velocity component are shown in Figs. 14(a)-14(1). Like the streamwise component, the largest velocity fluctuations in the lower-order eigenmodes typically occur in the reattachment region, however the magnitudes are significantly less than those observed in the streamwise component. Nevertheless, it is clear, and important to emphasize, that all near wake regions undergo a significant motion in both the streamwise and vertical directions. The alternating distribution of vertical velocity fluctuations at Mach 1.46 substantiates the presence of a predominant sinuous mode. This flapping motion can be clearly seen to decrease with increasing Mach number, as evidenced by the vortex shedding process downstream of reattachment becoming significantly weaker. While at Mach 1.46 many of the higher-order eigenmodes typically show the same fundamental features as their lowerorder counterparts, this is not generally the case at the higher
Mach numbers. The higher-order eigenmodes become increasingly difficult to interpret with increasing compressibility. This has also been reported in earlier work, which considered intermediate Mach numbers not considered here. ${ }^{31}$ As noted by Clemens and Mungal, ${ }^{21}$ numerical studies based upon linear stability analyses have shown that at low compressibility $\left(M_{c}<0.6\right.$ say), the flow structure is dominated by Kelvin-Helmholtz instabilities. At higher convective Mach numbers, the dominant instabilities become oblique, and result in a more three-dimensional flow structure organization. It has therefore been proposed that structural coherence degeneration occurs with increasing compressibility, and can be attributed to the role of these three-dimensional instability modes. ${ }^{21,56}$ This may provide a possible explanation for the difficulty in interpreting the present higher-order eigenmodes with increasing compressibility, since the results are based upon a two-dimensional representation of an essentially three-dimensional flowfield. It should be remarked, however, that in the present experiments, there were increased difficulties associated with optical diagnostics with increasing Mach number; namely, flow seeding of an increasingly smaller wake of lower density ratio.

In order to further clarify the observations made above, projections of several of the low-order eigenmodes onto their mean flow are made. Recall from Eq. (8) that one can arbitrarily choose a finite number $K$ of the most energetic modes to form a subspace spanned by the first $K$ eigenmodes. Similarly, subspaces can also be formulated based on a single eigenmode, by first ordering the temporal coefficients of all $M$ observations, such that $a_{k}\left(t_{n}\right) \leqslant a_{k}\left(t_{n+1}\right) \leqslant \cdots \leqslant a_{k}\left(t_{M}\right)$. An eigenmode can then be projected onto the mean velocity field to yield $M$ subspaces, the $n$th subspace of the $k$ th eigenmode $u_{n}^{k}(x, y)$ given by

$$
u_{n}^{k}(x, y)=\bar{u}(x, y)+a_{k}\left(t_{n}\right) \psi_{k}(x, y), \quad n=1, \ldots, M .
$$

These subspaces provide a convenient method to analyze the dynamical behavior given by the $k$ th eigenmode. The motion of the reattachment point contained within these subspaces (determined from the streamline topology) was chosen for comparison. The subspace reattachment point is defined by the streamwise and vertical coordinates $x_{r}$ and $y_{r}$, respectively, and we arbitrarily define a reattachment point angle $\theta$ from the wake axis,

$$
\theta=\tan ^{-1}\left(\frac{y_{r}}{x_{r}}\right)
$$

Note that $M$ data points are available for a given eigenmode, but only a sample of these results showing 1 in 30 data points is displayed for clarity. Irregularities in the data are due to the uncertainty in accurately defining the reattachment point within a subspace.

A polar plot of the subspace reattachment point trajectory for the first eigenmode at Mach 1.46 is shown in Fig. 15. Generally, it can be seen that both an appreciable streamwise and vertical deviation of the reattachment point from its mean position (located at the origin) occurs. The first eigenmode of the Mach 1.46 case reveals that a significant vertical motion of the reattachment point occurs, consistent with the 


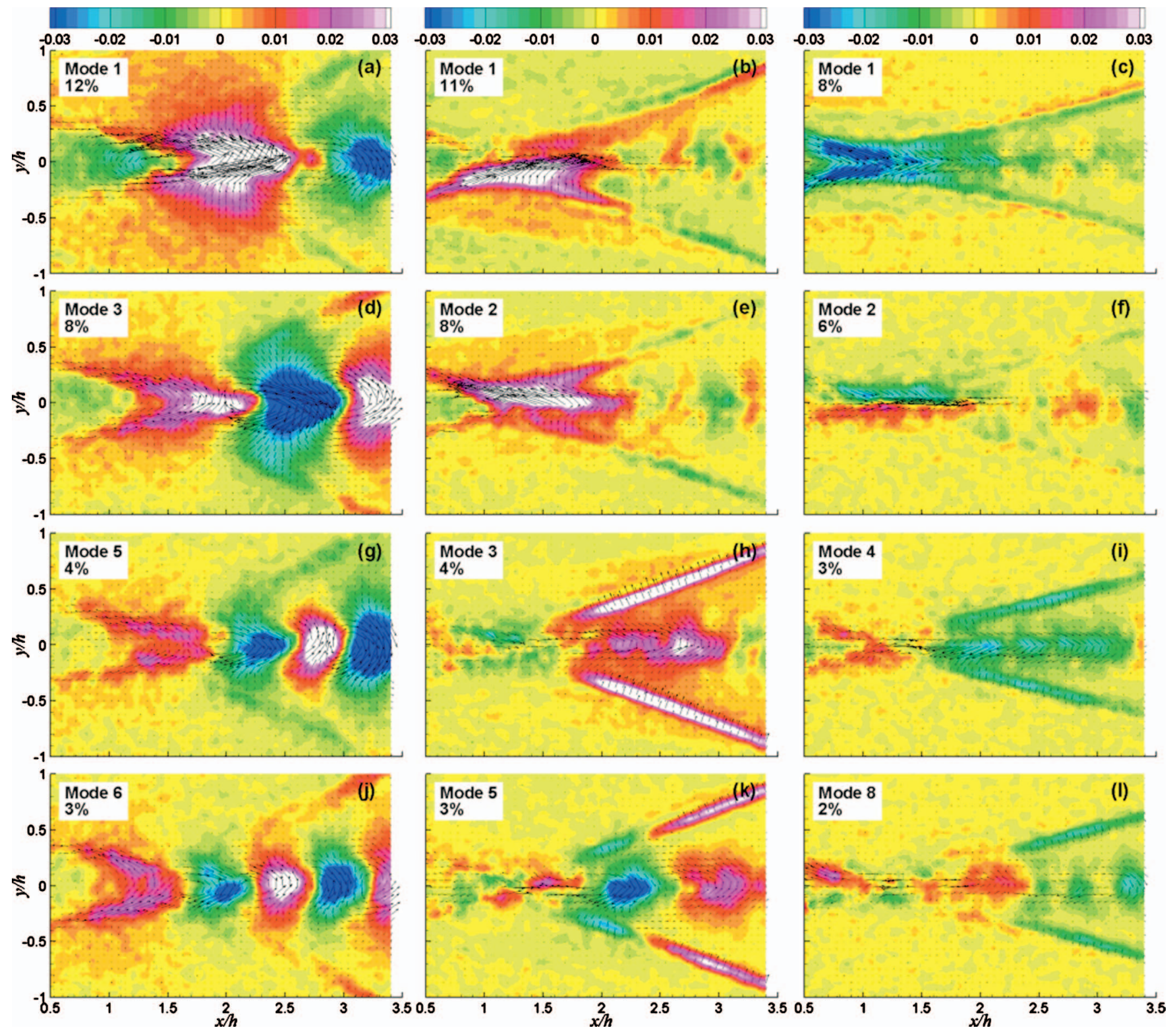

FIG. 14. (Color) POD eigenmodes: vertical velocity component $v^{\prime} / U_{\infty}$ : (left) Mach 1.46, (center) Mach 1.78, (right) Mach 2.27. Mode number and energy content shown (inset).

observations made in the PIV results, and supports the claim that the predominant global mode is a sinuous flapping motion. The first two eigenmodes of the Mach 1.78 case are shown in Fig. 16. These two reattachment trajectories are symmetrically arranged about the wake axis, and reflect the symmetry observed between the two eigenmodes. These results support the claim that the predominant global mode consists of both a significant sinuous and pulsating motionthe predominant global modes of the Mach 1.46 and 2.27 cases, respectively. In contrast, the Mach 2.27 case, shown in Fig. 17, appears to lack eigenmode pairing. A significant streamwise motion of the reattachment point can be observed in the second eigenmode of the Mach 2.27 case. It is somewhat surprising to see that the corresponding first eigenmode does not exhibit the same behavior. It should be remarked, however, that the motion of the reattachment point itself does not necessarily give a clear indication of the underlying flow organization. A visual animation of the subspaces of this eigenmode reveals that it is in fact not dissimilar (in a global sense) to its higher-order counterpart. Such motion of the reattachment point and associated recompression shock wave system may have important consequences for the fluctuating base pressure, as well as fluctuating loads on the body itself.

It is worth noting that attempts were made to represent a coherent, phase-resolved component, by considering the correlation of the temporal coefficients of the eigenmodes that appeared to be in phase-quadrature. This approach has proven successful in characterizing the vortex shedding process in low-speed near wakes. ${ }^{54}$ No significant correlation could be found, however, between any of the temporal coefficients considered in the present study. This is thought to be the result of variations in the cyclic vortex shedding process induced by the small-scale velocity fluctuations, or turbulence within the flow, as well as the presence of random 


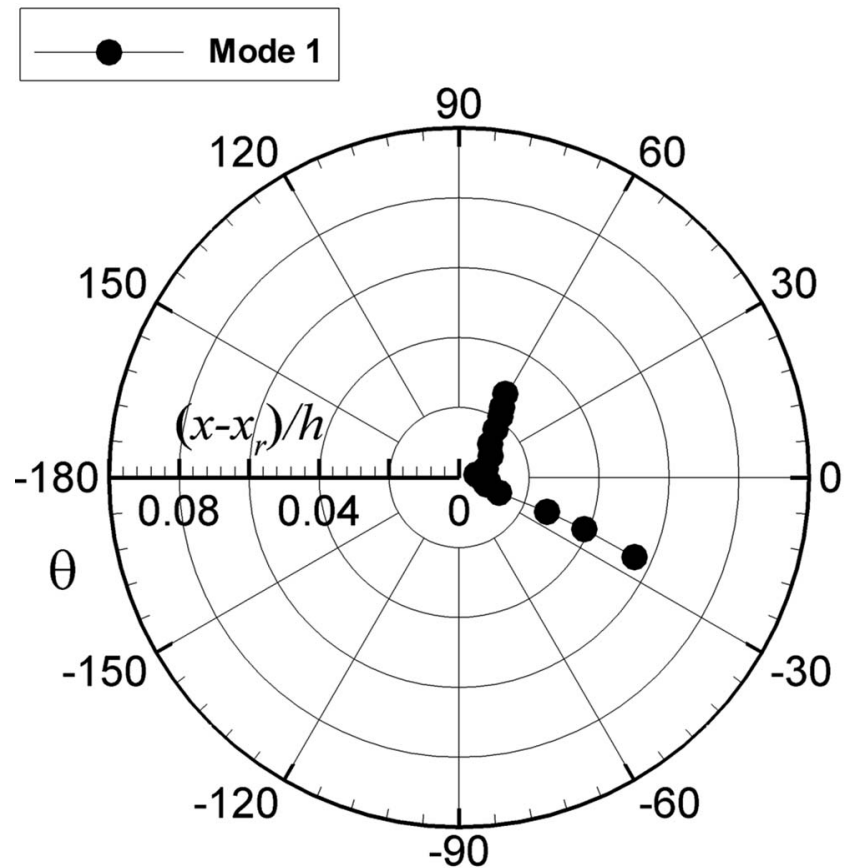

FIG. 15. Polar plot of the eigenmode subspace reattachment point trajectory at Mach 1.46.

noise and occasional poor data quality associated with the experimental data. The eigenmode energy distributions support this statement, since small-scale velocity fluctuations are present in higher-order eigenmodes, which are still generally associated with an appreciable fraction of the total kinetic energy.

Overall, it would appear that the eigenmodes presented above, along with the instantaneous velocity fields, describe

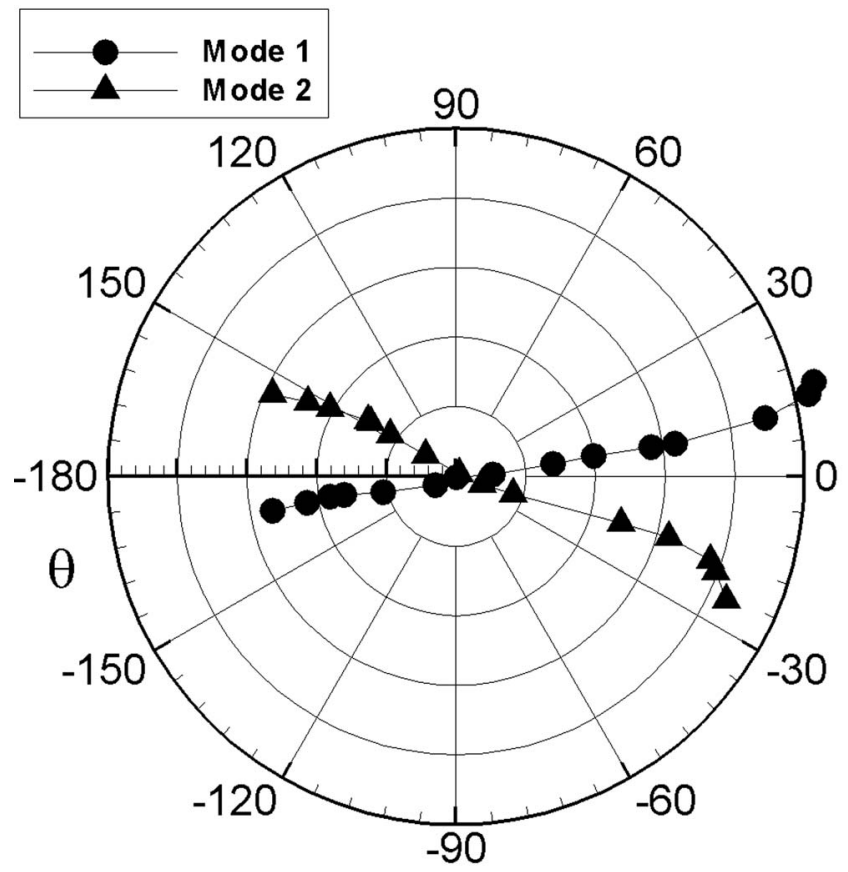

FIG. 16. Polar plot of the eigenmode subspace reattachment point trajectory at Mach 1.78.

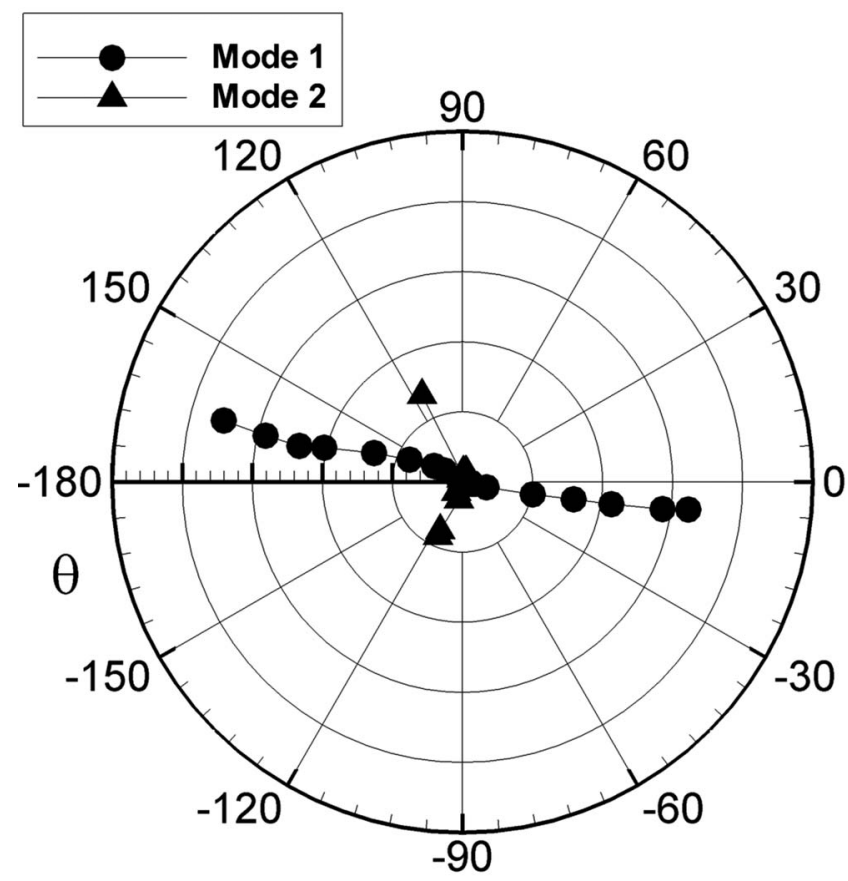

FIG. 17. Polar plot of the eigenmode subspace reattachment point trajectory at Mach 2.27.

an evolution of the predominant global mode with compressibility, within the Mach number range considered. The predominant flow mechanisms responsible for the fluctuating characteristics of transonic and supersonic base flows have been explored by Kawai ${ }^{11}$ in his systematic LES/RANS studies of subsonic, transonic, and supersonic cylindrical base flows. Under transonic conditions, they are thought to be associated with the close relation between unsteady vortex shedding induced by the instability of the shear layers and the local shock waves, which oscillate along the axial direction of the free shear layers. Under fully supersonic conditions, the predominant flow mechanisms are thought to be based upon the oscillation of the free shear layers and the recompression shock waves. The evolution of these two modes has been further hypothesized to be associated with the changes that occur in the characteristics of free shear layers with increasing compressibility; namely, from a Kelvin-Helmholtz type of instability at subsonic speeds, to a coupling between multiple waves at supersonic speeds, as a result of a possible feedback loop from the oscillation of the recompression shock waves. Clearly, further research needs to be conducted in this area to help unify experimental and computational efforts concerning both planar and axisymmetric base flows. It is interesting to note that the flapping motion reported in the present study has in fact been proposed to help explain the substantial time variation of base pressure fluctuations commonly observed in supersonic base flow experiments. ${ }^{14}$

\section{CONCLUSIONS}

The unsteady flow features of compressible, twodimensional, planar base flows have been studied. Experiments were performed under a range of low-supersonic con- 
ditions in order to gain a better understanding of the effects of compressibility on the organized global dynamics, and their role in the distributed nature of the turbulence properties. PIV was used as the primary diagnostic tool in combination with data processing using POD. Mean and instantaneous velocity measurements enabled a visualization of the near wake behavior. Turbulence statistics were then presented, and the role of organized global dynamics in the distributed nature of the turbulence properties was addressed. The data were then used in combination with POD to make further statements regarding the unsteady flow organization. From these data, the following conclusions can be drawn:

A predominant global mode is present in each test case considered, determined both by visual inspection of the PIV results and confirmed by the POD eigenmodes. These predominant global modes undergo an evolution with compressibility, within the Mach number range considered. At Mach 1.46, both the PIV results and the eigenmodes show a fluctuating velocity pattern, indicative of the train of quasistreamwise vortices associated with a convected vortex street. The predominant global mode can therefore be characterized by a sinuous flapping motion. With increasing Mach number, this sinuous mode decreases in amplitude. The eigenmodes show streamwise velocity fluctuations becoming increasingly elongated, and the predominant global mode evolves into a pulsating motion aligned with the wake axis at Mach 2.27. The predominant global mode of the intermediate case of Mach 1.78 consists of both the global modes of the Mach 1.46 and 2.27 cases. All eigenmodes show very little flow activity in the recirculating flow region.

The predominant global modes have a significant effect on the motion of the recompression shock wave system. The sinuous mode is typically associated with an alternating translating motion of the recompression shock waves, whereas the pulsating mode is typically associated with the translation of both recompression shock waves together. A region of expansion where the redeveloping wake becomes locally convex often occurs, and influences the overall compression that takes place in the outer flow.

The predominant global modes play an important role in the distributed nature of the turbulence properties. The turbulent mixing processes become increasingly confined to a narrower wake with increasing compressibility. Global maximum levels of the streamwise turbulence intensity and the kinematic Reynolds shear stress occur within the vicinity of the mean reattachment location, and show no systematic trend with compressibility. In contrast, the maximum vertical turbulence intensity moves systematically upstream, from the redeveloping wake, toward the mean reattachment location. The vertical turbulence intensity decays thereafter more slowly than the other turbulence quantities. Overall, the local maximum levels of the turbulence properties decrease appreciably with increasing compressibility.

\section{ACKNOWLEDGMENT}

This work is supported by the Dutch Technology Foundation STW under the VIDI-Innovation Impulse program, Grant No. DLR.6198.
${ }^{1}$ N. T. Clemens, S. P. Petullo, and D. S. Dolling, "Large-scale structure evolution in supersonic interacting shear layers," AIAA J. 34, 2062 (1996).

${ }^{2}$ M. Samimy, M. F. Reeder, and G. S. Elliot, "Compressibility effects on large structures in free shear flows," Phys. Fluids A 4, 1251 (1992).

${ }^{3}$ K. M. Smith and J. C. Dutton, "Investigation of large-scale structures in supersonic planar base flows," AIAA J. 34, 1146 (1996).

${ }^{4}$ C. J. Bourdon and J. C. Dutton, "Planar visualizations of large-scale turbulent structures in axisymmetric supersonic separated flows," Phys. Fluids 11, 201 (1999).

${ }^{5}$ A. Amatucci, J. C. Dutton, D. W. Kuntz, and A. L. Addy, "Two-stream, supersonic, wake flowfield behind a thick base. Part I: General features," AIAA J. 30, 2039 (1992).

${ }^{6}$ F. Scarano and B. W. van Oudheusden, "Planar velocity measurements of a two-dimensional compressible wake," Exp. Fluids 34, 430 (2003).

${ }^{7}$ M. Samimy and A. L. Addy, "Interaction between two compressible, turbulent free shear layers," AIAA J. 24, 1918 (1986).

${ }^{8}$ J. L. Herrin and J. C. Dutton, "The turbulence structure of a reattaching axisymmetric compressible free shear layer," Phys. Fluids 9, 3502 (1997).

${ }^{9}$ B. A. Boswell and J. C. Dutton, "Shear layer development in a threedimensional compressible base flow," Phys. Fluids 16, 3031 (2004).

${ }^{10}$ J. L. Herrin and J. C. Dutton, "Effect of a rapid expansion on the development of compressible free shear layers," Phys. Fluids 7, 159 (1995).

${ }^{11}$ S. Kawai, "Computational analysis of the characteristics of high speed base flows," Ph.D. dissertation, University of Tokyo, Tokyo (2005).

${ }^{12} \mathrm{C}$. Fureby and M. Kupiainen, "Large eddy simulation of supersonic axisymmetrc baseflow," in Turbulent Shear Flow Phenomena, edited by N. Kasagi, J. K. Eaton, R. Friedrichs, J. A. C. Humphrey, M. A. Leschziner, and T. Miyauchi (Sendai, Japan, 2003).

${ }^{13}$ F. Simon, S. Deck, P. Guillen, and P. Sagaut, "Reynolds-averaged NavierStokes/large-eddy simulations of supersonic base flow," AIAA J. 44, 2578 (2006).

${ }^{14}$ J. R. Janssen and J. C. Dutton, "Time-series analysis of supersonic basepressure fluctuations," AIAA J. 42, 605 (2004).

${ }^{15}$ D. Hunt and D. A. Nixon, "A very large eddy simulation of an unsteady shock wave/turbulent boundary layer interaction," 26th AIAA Fluid Dynamics Conference (AIAA, San Diego, CA, 1995).

${ }^{16} \mathrm{M}$. Raffel, C. Willert, and J. Kompenhans, Particle Image Velocimetry: A Practical Guide (Springer-Verlag, Berlin, 1998).

${ }^{17}$ A. Krothapalli, D. P. Wishart, and L. M. Lourenco, "Near field structure of a supersonic jet: Online PIV study," 7th International Symposium on the Application of Laser Techniques to Fluid Mechanics, Lisbon, Portugal (1994).

${ }^{18}$ W. D. Urban and M. G. Mungal, "Planar velocity measurements in compressible mixing layers," J. Fluid Mech. 431, 189 (2001).

${ }^{19}$ B. Ganapathisubrami, N. T. Clemens, and D. S. Dolling, "Large-scale motions in a supersonic turbulent boundary layer," J. Fluid Mech. 556, 271 (2006).

${ }^{20}$ D. W. Bogdanoff, "Compressibility effects in turbulent shear layers," AIAA J. 21, 926 (1983).

${ }^{21}$ N. T. Clemens and M. C. Mungal, "Two- and three-dimensional effects in the supersonic mixing layer," AIAA J. 30, 973 (1992).

${ }^{22}$ S. G. Goebel and J. C. Dutton, "Experimental study of compressible turbulent mixing layers," AIAA J. 29, 538 (1991).

${ }^{23}$ G. S. Elliot and M. Samimy, "Compressibility effects in free shear layers," Phys. Fluids A 2, 1231 (1990).

${ }^{24}$ N. L. Messersmith and J. C. Dutton, "Characteristic features of large structures in compressible mixing layers," AIAA J. 34, 1814 (1996).

${ }^{25}$ J. L. Lumley, "The structure of inhomogeneous turbulent flows," in Atmospheric Turbulence and Radio Wave Propagation, edited by A. M. Yaglom (Nauka, Moscow, 1967).

${ }^{26}$ A. Liberzon, R. Gurka, I. Tiselj, and R. Hetsroni, "Spatial characterization of the numerically simulated vorticity fields of a flow in a flume," Theor. Comput. Fluid Dyn. 19, 115 (2005).

${ }^{27}$ S. Maurel, J. Boree, and J. L. Lumley, "Extended proper orthogonal decomposition: Application to jet/vortex interaction," Flow, Turbul. Combust. 67, 125 (2001).

${ }^{28}$ D. Moreno, A. Krothapalli, M. B. Alkislar, and L. M. Lourenco, "Lowdimensional model of a supersonic rectangular jet," Phys. Rev. E 69, 026304 (2004).

${ }^{29}$ M. J. Molezzi and J. C. Dutton, "Study of subsonic base cavity flowfield structure using particle image velocimetry," AIAA J. 33, 201 (1995).

${ }^{30}$ M. J. Molezzi and J. C. Dutton, "Application of particle image velocimetry in high-speed separated flows," AIAA J. 31, 438 (1993). 
${ }^{31}$ R. A. Humble, F. Scarano, and B. W. van Oudheusden, "Unsteady planar base flow investigation using particle image velocimetry and proper orthogonal decomposition," 44th AIAA Aerospace Sciences Meeting and Exhibit (AIAA, Reno, NV, 2006).

${ }^{32}$ S. L. Gai, D. P. Hughes, and M. S. Perry, "Large-scale structures and growth of a flat plate compressible wake," AIAA J. 40, 1164 (2002).

${ }^{33} \mathrm{~W}$. Althaus, "Experimental investigation of vortex formation in the wake of a flat plate for subsonic and supersonic freestream Mach numbers," Exp. Fluids 9, 267 (1990).

${ }^{34}$ D. Papamoschou and A. Bunyajitradulya, "Evolution of large eddies in compressible shear layers," Phys. Fluids 9, 756 (1997).

${ }^{35}$ L. H. Benedict and R. D. Gould, "Towards better uncertainty estimates for turbulence statistics," Exp. Fluids 22, 129 (1996).

${ }^{36}$ F. Scarano, "Iterative image deformation methods in PIV," Meas. Sci. Technol. 13, R1 (2002).

${ }^{37}$ P. Holmes, J. L. Lumley, and G. Berkooz, Turbulence, Coherent Structures, Dynamical Systems and Symmetry (Cambridge University Press, Cambridge, UK, 1996).

${ }^{38} \mathrm{~L}$. Sirovich, "Turbulence and the dynamics of coherent structures," Q Appl. Math. 45, 561 (1987).

${ }^{39}$ B. Patte-Rouland, G. Lalizel, J. Moreau, and E. Rouland, "Flow analysis of an annular jet by particle image velocimetry and proper orthogonal decomposition," Meas. Sci. Technol. 12, 1404 (2001).

${ }^{40}$ C. J. Bourdon and J. C. Dutton, "Shear layer flapping and interface convolution in a separated supersonic flow," 30th AIAA Fluid Dynamics Conference (AIAA, Norfolk, VA, 1999).

${ }^{41}$ N. T. Clemens and M. G. Mungal, "Large-scale structure and entrainment in the supersonic mixing layer," J. Fluid Mech. 284, 171 (1995).

${ }^{42}$ A. L. Kastengren, J. C. Dutton, and G. S. Elliot, "Reattachment shock unsteadiness in supersonic blunt-base cylinder wakes," 43rd AIAA Aerospace Sciences Meeting and Exhibit (AIAA, Reno, NV, 2005).

${ }^{43}$ H. L. Petrie, M. Samimy, and A. L. Addy, "Compressible separated flows," AIAA J. 24, 1971 (1986).

${ }^{44}$ P. Ardonceau, D. H. Lee, T. Alziary de Roquefort, and R. Goethals, "Turbulence behaviour in a shock-wave/turbulent boundary layer interaction," AGARD CP-271, Paper No. 8 (1980).

${ }^{45}$ R. Friedrich, H. Foysi, and J. Sesterhenn, "Turbulent momentum and passive scalar transport in supersonic channel flow," J. Braz. Soc. Mech. Sci. XXVIII, 174 (2006).

${ }^{46}$ J. Délery and J. G. Marvin, "Shock-wave boundary layer interactions," AGARDograph 280 (1986).

${ }^{47}$ M. Samimy, H. L. Petrie, and A. L. Addy, "A study of compressible turbulent reattaching free shear layers," AIAA J. 24, 261 (1986).

${ }^{48}$ J. K. Eaton and J. P. Johnston, "A review of research on subsonic turbulent flow reattachment," AIAA J. 19, 1093 (1981).

${ }^{49}$ D. Papamoschou and A. Roshko, "Observations of supersonic free shear layers," 24th Aerospace Sciences Meeting and Exhibit (AIAA, Reno, NV, 1986).

${ }^{50}$ M. Xia and G. Karniadakis, "A low-dimensional model for simulating three-dimensional cylinder flow," J. Fluid Mech. 458, 181 (2002).

${ }^{51}$ J. Kirby, J. Boris, and L. Sirovich, "An eigenfunction analysis of axisymmetric jet flow," J. Comput. Phys. 90, 98 (1990).

${ }^{52}$ J. Kostas, J. Soria, and M. S. Chong, "A comparison between snapshot POD analysis of PIV velocity and vorticity data," Exp. Fluids 38, 146 (2005).

${ }^{53}$ K. Hayakawa, A. J. Smits, and S. M. Bogdonoff, "Turbulence measurements in a compressible reattaching shear layer," AIAA J. 22, 889 (1984).

${ }^{54}$ B. W. van Oudheusden, F. Scarano, N. P. van Hinsberg, and D. W. Watt, "Phase-resolved characterization of vortex shedding in the near wake of a square-section cylinder at incidence," Exp. Fluids 39, 86 (2005).

${ }^{55} \mathrm{H}$. Kemmoun, "Unsteady measurements in supersonic base flow," Masters thesis, Delft University of Technology, Delft, The Netherlands (2002).

${ }^{56}$ N. D. Sandham and W. C. Reynolds, "Compressible mixing layer theory: Linear theory and direct simulation," AIAA J. 28, 618 (1990). 